\title{
HATS-6b: A WARM SATURN TRANSITING AN EARLY M DWARF STAR, AND A SET OF EMPIRICAL RELATIONS FOR CHARACTERIZING K AND M DWARF PLANET HOSTS*
}

\author{
J. D. Hartman ${ }^{1}$, D. Bayliss ${ }^{2}$, R. Brahm ${ }^{3,4}$, G. Á. Bakos ${ }^{1,11,12}$, L. Mancini ${ }^{5}$, A. Jordán ${ }^{3,4}$, K. Pennev ${ }^{1}$,

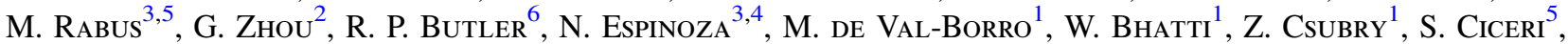 \\ T. Henning ${ }^{5}$, B. Schmidt ${ }^{2}$, P. Arriagada ${ }^{6}$, S. Shectman ${ }^{7}$, J. Crane ${ }^{7}$, I. Thompson ${ }^{7}$, V. Suc ${ }^{3}$, B. Csák ${ }^{5}$,

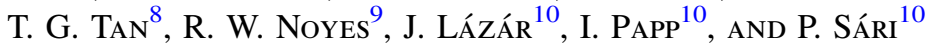 \\ ${ }^{1}$ Department of Astrophysical Sciences, Princeton University, NJ 08544, USA; jhartman@ astro.princeton.edu \\ ${ }^{2}$ The Australian National University, Canberra, Australia \\ ${ }^{3}$ Instituto de Astrofísica, Facultad de Física, Pontificia Universidad Católica de Chile, Av.Vicuña Mackenna 4860, 7820436 Macul, Santiago, Chile \\ ${ }^{4}$ Millennium Institute of Astrophysics, Av.Vicuña Mackenna 4860, 7820436 Macul, Santiago, Chile \\ ${ }^{5}$ Max Planck Institute for Astronomy, Heidelberg, Germany \\ ${ }^{6}$ Department of Terrestrial Magnetism, Carnegie Institution of Washington, 5241 Broad Branch Road NW, Washington, DC 20015-1305, USA \\ ${ }^{7}$ The Observatories of the Carnegie Institution of Washington, 813 Santa Barbara Street, Pasadena, CA 91101, USA \\ ${ }^{8}$ Perth Exoplanet Survey Telescope, Perth, Australia \\ ${ }^{9}$ Harvard-Smithsonian Center for Astrophysics, Cambridge, MA, USA \\ ${ }^{10}$ Hungarian Astronomical Association, Budapest, Hungary \\ Received 2014 August 8; accepted 2015 February 13; published 2015 April 28
}

\begin{abstract}
We report the discovery by the HATSouth survey of HATS-6b, an extrasolar planet transiting a $V=15.2 \mathrm{mag}$, $i=13.7 \mathrm{mag}$ M1V star with a mass of $0.57 M_{\odot}$ and a radius of $0.57 R_{\odot}$. HATS-6b has a period of $P=3.3253 \mathrm{~d}$, mass of $M_{p}=0.32 M_{J}$, radius of $R_{p}=1.00 R_{J}$, and zero-albedo equilibrium temperature of $T_{\mathrm{eq}}=712.8 \pm 5.1 \mathrm{~K}$. HATS-6 is one of the lowest mass stars known to host a close-in gas giant planet, and its transits are among the deepest of any known transiting planet system. We discuss the follow-up opportunities afforded by this system, noting that despite the faintness of the host star, it is expected to have the highest $K$-band $\mathrm{S} / \mathrm{N}$ transmission spectrum among known gas giant planets with $T_{\mathrm{eq}}<750 \mathrm{~K}$. In order to characterize the star we present a new set of empirical relations between the density, radius, mass, bolometric magnitude, and $\mathrm{V}-, J_{-}, H$ - and $\mathrm{K}$-band bolometric corrections for main sequence stars with $M<0.80 M_{\odot}$, or spectral types later than K5. These relations are calibrated using eclipsing binary components as well as members of resolved binary systems. We account for intrinsic scatter in the relations in a self-consistent manner. We show that from the transit-based stellar density alone it is possible to measure the mass and radius of a $\sim 0.6 M_{\odot}$ star to $\sim 7$ and $\sim 2 \%$ precision, respectively. Incorporating additional information, such as the $V-K$ color, or an absolute magnitude, allows the precision to be improved by up to a factor of two.
\end{abstract}

Key words: planetary systems - stars: individual (HATS-6) - techniques: photometric - techniques: spectroscopic

Supporting material: machine-readable and VO tables

\section{INTRODUCTION}

One of the goals in the study of exoplanetary systems is to determine how the properties of planets depend on the properties of their host stars. An important parameter in this respect is the host star mass. Results from both radial velocity and transit surveys indicate that the occurrence rate of gas giant planets is a steep function of stellar mass scaling approximately as $N \propto M_{\star}$ (Johnson et al. 2010) for main sequence stars of type $\mathrm{M}$ through $\mathrm{F}$ (smaller planets, on the other hand, appear to be more frequent around $\mathrm{M}$ dwarfs than around hotter stars;

\footnotetext{
* The HATSouth network is operated by a collaboration consisting of Princeton University (PU), the Max Planck Institute für Astronomie (MPIA), the Australian National University (ANU), and the Pontificia Universidad Católica de Chile (PUC). The station at Las Campanas Observatory (LCO) of the Carnegie Institute is operated by PU in conjunction with PUC, the station at the High Energy Spectroscopic Survey (H.E.S.S.) site is operated in conjunction with MPIA, and the station at Siding Spring Observatory (SSO) is operated jointly with ANU. This paper includes data gathered with the $6.5 \mathrm{~m}$ Magellan Telescopes located as Las Campanas Observatory, Chile. Based in part on observations made with the MPG $2.2 \mathrm{~m}$ Telescope and the ESO $3.6 \mathrm{~m}$ Telescope at the ESO Observatory in La Silla. This paper uses observations obtained with facilities of the Las Cumbres Observatory Global Telescope.

${ }^{11}$ Alfred P. Sloan Research Fellow.

12 Packard Fellow.
}

Howard et al. 2012). The low occurrence rate of these planets around $\mathrm{M}$ dwarfs, coupled with the fact that most surveys primarily target FGK dwarf stars, means that only one hot Jupiter has so far been discovered around an M0 dwarf (Kepler-45; Johnson et al. 2012), and only a handful of others have been found around very late K dwarf stars (WASP-80, Triaud et al. 2013b; WASP-43, Hellier et al. 2011; and HAT-P54, Bakos et al. 2014, being the only three known transitinghot-Jupiter-hosting $\mathrm{K}$ dwarfs with $M<0.65 M_{\odot}$ ).

In addition to enabling the study of planetary properties as a function of stellar mass, finding planets around late-type stars has at least two other advantages. The small sizes of these stars coupled with their low luminosities means that a planet with a given radius and orbital period around a late-type star will produce deeper transits and have a cooler equilibrium temperature than if it were around a larger star. This makes planets around late-type stars attractive targets for carrying out detailed follow-up observations, such as atmospheric characterizations. A second, perhaps underappreciated, advantage of these stars is that they are remarkably simple in their bulk properties. Whereas, due to stellar evolution, the radius of a solar-metallicity, solar-mass star varies by $\sim 40 \%$ over the $10 \mathrm{Gyr}$ age of the Galactic disk, the radius of a $0.6 M_{\odot}$ star 
varies by less than $\sim 5 \%$ over the same time span. The parameters of low-mass stars follow tight main-sequence relations, enabling high-precision measurements of their properties from only a single (or a few) observable(s). The precision of the stellar parameters feeds directly into the precision of the planetary parameters, so that in principle planets around low-mass stars may be characterized with higher precision than those around higher mass stars.

Here we present the discovery of a transiting, short-period, gas-giant planet around an M1 dwarf star. This planet, HATS$6 \mathrm{~b}$, was discovered by the HATSouth survey, a global network of fully automated wide-field photometric instruments searching for transiting planets (Bakos et al. 2013). HATSouth uses larger-diameter optics than other similar projects allowing an enhanced sensitivity to faint $\mathrm{K}$ and $\mathrm{M}$ dwarfs.

We also present a new set of empirical relations to use in characterizing the properties of transiting planet-host stars with $M<0.80 M_{\odot}$. While there has been much discussion in the literature of the apparent discrepancy between observations and various theoretical models for these stars (e.g., Torres \& Ribas 2002; Ribas 2003; Torres 2013; Zhou et al. 2014, and references therein), the growing set of well-characterized lowmass stars has revealed that, as expected, these stars do follow tight main sequence relations. We show that from the bulk density of the star alone, which is determined from the transit light curve and RV observations, it is possible to measure the mass and radius of a $\sim 0.6 M_{\odot}$ star to $\sim 7 \%$ and $\sim 2 \%$ precision, respectively. Incorporating additional information, such as the $V-K$ color, or an absolute magnitude, allows the precision to be improved by a factor of two.

The layout of the paper is as follows. In Section 2 we report the detection of the photometric signal and the follow-up spectroscopic and photometric observations of HATS-6. In Section 3 we describe the analysis of the data, beginning with ruling out false positive scenarios, continuing with our global modeling of the photometry and radial velocities, and finishing with the determination of the stellar parameters and the planetary parameters that depend on them, using both theoretical stellar models as well as the empirical relations that we derive here. Our findings are discussed in Section 4.

\section{OBSERVATIONS}

\subsection{Photometric Detection}

Observations of a field containing HATS-6 (see Table 9 for identifying information) were carried out with the HS-2, HS-4, and HS-6 units of the HATSouth network (located at Las Campanas Observatory in Chile, the High Energy Spectroscopic Survey gamma-ray telescope site in Namibia, and Siding Spring Observatory in Australia, respectively; see Bakos et al. 2013 for a detailed description of the HATSouth network) between UT 2009 September 17 and UT 2010 September 10. A total of 5695, 5544, and 88 images included in our final trend and outlier-filtered light curves were obtained with HS-2, HS-4, and HS-6, respectively. Observations were made through a Sloan $r$ filter, using an exposure time of $240 \mathrm{~s}$ and a median cadence of $293 \mathrm{~s}$ ( see also Table 1 ).

The data were reduced to trend-filtered light curves following Bakos et al. (2013). We apply two empirical trend filtering techniques to the data. The first is to decorrelate the light curves against a set of measured parameters that vary from image to image, including the $X$ and $Y$ sub-pixel coordinates of the star, three parameters describing the shape of the image point-spread function, the hour angle of the observations, the zenith distance, and the sky background near the target. We refer to this filtering as External Parameter Decorrelation (EPD; Bakos et al. 2010). The second filtering technique is to use the Trend Filtering Algorithm (TFA) due to Kovács et al. (2005). In this method we select a list of $\sim 800$ template light curves uniformly distributed in position across the field and coming from stars with a broad range of magnitudes. We then fit the EPD-filtered light curve for HATS-6 as a linear combination of these $\sim 800$ templates and subtract the fit from the observations. The filtered light curve for HATS-6 has a point-to-point rms of $0.02 \mathrm{mag}$ and is dominated by noise from the background sky. Transits were identified in the resulting HATSouth light curve of HATS-6 using the Box Least Squares (BLS; Kovács et al. 2002) algorithm. Figure 1 shows the phase-folded HATSouth light curve of HATS-6 together with our best-fit transit model, while the photometric measurements are provided in Table 2 .

We searched the residual HATSouth light curve for additional transit signals using BLS but found no significant detection. We also searched for continuous quasi-periodic variability using both the Discrete Fourier Transform (Kurtz 1985) and the Discrete Autocorrelation Function (Edelson \& Krolik 1988). We find a possible signal in the pre-TFA light curve with a period of $P=35.1$ days and a signal-to-noise ratio $(\mathrm{S} / \mathrm{N})$, measured in the power spectrum, of 12.3. This signal is not seen in the autocorrelation function, nor is it seen in the light curve after processing with TFA. While potentially due to stellar rotation, this may also be timecorrelated noise. We therefore do not claim a measurement of the photometric rotation period for this star.

\subsection{Photometric Follow-up}

In order to confirm the transit signal and determine the parameters of the system with better accuracy, we carried out follow-up photometric observations of HATS-6 using the $0.3 \mathrm{~m}$ Perth Exoplanet Survey Telescope (PEST), the CTIO $0.9 \mathrm{~m}$, telescopes in the LCOGT $1 \mathrm{~m}$ network (Brown et al. 2013), and GROND on the MPG $2.2 \mathrm{~m}$ (Greiner et al. 2008). Key aspects of these observations, including the dates of the observations, the number of images obtained, the cadence, filter used, and the precision of the resulting HATS- 6 light curves, as measured from the rms of the residuals from our best-fit model, are summarized in Table 1. The light curves are plotted in Figures 2 and 3, while the measurements are provided in Table 2. Details regarding the PEST instrument, our observational procedure, as well as our reduction and photometry methods can be found in Zhou et al. (2014; see Penev et al. 2013 and Mohler-Fischer et al. 2013) for similar information regarding the GROND observations. Because this is the first time we have used the CTIO $0.9 \mathrm{~m}$ and the LCOGT $1 \mathrm{~m}$ network, we describe our use of these facilities in more detail below.

On the nights of 2012 September 3 and 2013 October 27, we performed photometric observations of HATS- 6 using the CTIO $0.9 \mathrm{~m}$ telescope, which has a CCD with a 13 ." $6 \times 13$. $^{\prime \prime} 6$ field of view (FOV). On the first night we used a Gunn $z^{\prime}$ filter and on the latter one a Kron-Cousins $R_{C}$ filter. We defocused the telescope in order to broaden the point-spread function. Images from both nights were calibrated (bias subtracted and flat fielded) with custom Python routines. A fringing effect was 
Table 1

Summary of Photometric Observations

\begin{tabular}{|c|c|c|c|c|c|}
\hline Facility & Date(s) & Number of Images ${ }^{\mathrm{a}}$ & Cadence $(\mathrm{s})^{\mathrm{b}}$ & Filter & Precision (mmag) \\
\hline HS-2 & 2009 Sep-2010 Sep & 5695 & 295 & $r$ & 21.3 \\
\hline HS-4 & 2009 Sep-2010 Sep & 5544 & 293 & $r$ & 21.0 \\
\hline HS-6 & 2010 Aug-2010 Sep & 88 & 296 & $r$ & 18.6 \\
\hline PEST $0.3 \mathrm{~m}$ & 2013 Feb 17 & 58 & 205 & $R$ & 6.1 \\
\hline PEST $0.3 \mathrm{~m}$ & 2013 Feb 27 & 53 & 131 & $R$ & 5.6 \\
\hline PEST $0.3 \mathrm{~m}$ & 2013 Mar 29 & 24 & 261 & $R$ & 4.8 \\
\hline LCOGT $1 \mathrm{~m}$ & 2013 Dec 07 & 149 & 74 & $i$ & 3.8 \\
\hline GROND/MPG $2.2 \mathrm{~m}$ & 2014 Mar 06 & 95 & 155 & $g$ & 1.8 \\
\hline GROND/MPG $2.2 \mathrm{~m}$ & 2014 Mar 06 & 95 & 155 & $r$ & 1.1 \\
\hline GROND/MPG $2.2 \mathrm{~m}$ & 2014 Mar 06 & 95 & 155 & $i$ & 1.1 \\
\hline GROND/MPG $2.2 \mathrm{~m}$ & 2014 Mar 06 & 95 & 155 & $z$ & 1.1 \\
\hline
\end{tabular}

${ }^{a}$ Excludes images which were rejected as significant outliers in the fitting procedure.

${ }^{b}$ The mode time difference rounded to the nearest second between consecutive points in each light curve. Due to visibility, weather, pauses for focusing, etc., none of the light curves have perfectly uniform time sampling.
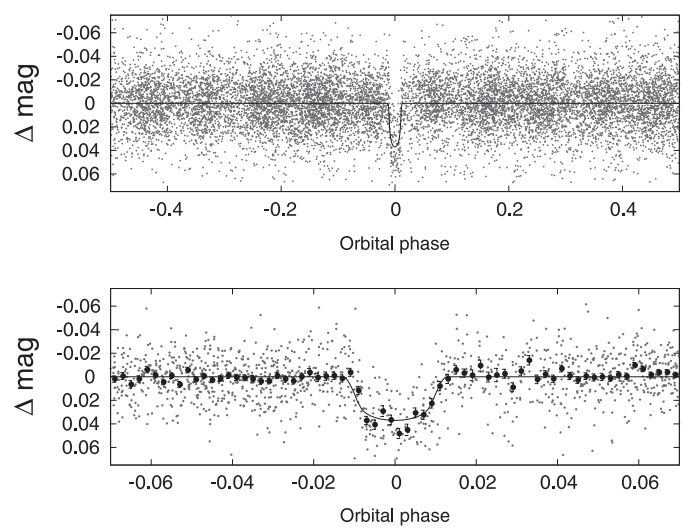

Figure 1. Unbinned instrumental $r$-band light curve of HATS-6 folded with the period $P=3.3252725$ days resulting from the global fit described in Section 3 . The solid line shows the best-fit transit model (see Section 3). In the lower panel we zoom-in on the transit; the dark filled points here show the light curve binned in phase using a bin-size of 0.002 .

seen in the images taken during our full moon night in the $z$ band. However, images taken with the $R_{C}$ filter did not show fringing. Therefore, we also took 53 empty field regions with the Gunn $z^{\prime}$ filter during our 2012 September CTIO run. We combined these images and subtracted the sky background and then scaled the combined image to match and remove the additive fringing effect seen in the science images. To reduce the calibrated images to light curves, we chose a reference image and calculated the shift of all images with respect to the reference image. From the reference image we extracted the position of the stars. Following Deeg \& Doyle (2001), the time series photometry was generated from these observations using optimized aperture photometry that maximizes the $\mathrm{S} / \mathrm{N}$ for each star. For all images in one night we used three fixed apertures and chose these to be much larger than the typical point-spread function in order to minimize the impact of the time-variable point-spread functions. The resulting light curves of HATS-6 have precisions of 4.7 and $3.7 \mathrm{mmag}$ on the first and second nights, respectively.

Observations of HATS- 6 were carried out using the LCOGT $1 \mathrm{~m}$ network on the UT nights of 2013 November 23 and
Table 2

Differential Photometry of HATS-6

\begin{tabular}{lrcccc}
\hline \hline $\begin{array}{l}\text { BJD } \\
(2400000+)\end{array}$ & \multicolumn{1}{c}{ Mag $^{\mathrm{a}}$} & $\sigma_{\mathrm{Mag}}$ & $\begin{array}{c}\text { Mag } \\
(\text { orig })^{\mathrm{b}}\end{array}$ & Filter & Instrument \\
\hline 55185.60957 & -0.00387 & 0.01084 & $\cdots$ & $r$ & HS \\
55145.70640 & -0.07475 & 0.03251 & $\cdots$ & $r$ & HS \\
55095.82750 & -0.01258 & 0.01255 & $\cdots$ & $r$ & HS \\
55275.39406 & 0.02245 & 0.01229 & $\cdots$ & $r$ & HS \\
55195.58763 & -0.00021 & 0.01587 & $\cdots$ & $r$ & HS \\
55182.28728 & 0.00324 & 0.01174 & $\cdots$ & $r$ & HS \\
55145.70968 & -0.00768 & 0.02964 & $\cdots$ & $r$ & HS \\
55185.61296 & 0.02073 & 0.01078 & $\cdots$ & $r$ & HS \\
55095.83089 & -0.02280 & 0.01379 & $\cdots$ & $r$ & HS \\
55105.80688 & -0.02863 & 0.02168 & $\cdots$ & $r$ & HS \\
\hline
\end{tabular}

Notes. The data are also available on the HATSouth website at http://www.hatsouth.org.

${ }^{a}$ The out-of-transit level has been subtracted. For the HATSouth light curve (rows with "HS" in the Instrument column), these magnitudes have been detrended using the EPD and TFA procedures prior to fitting a transit model to the light curve. Primarily as a result of this detrending, but also due to blending from neighbors, the apparent HATSouth transit depth is somewhat shallower than that of the true depth in the Sloan $r$ filter (the apparent depth is $90 \%$ that of the true depth). For the follow-up light curves (rows with an instrument other than "HS") these magnitudes have been detrended with the EPD procedure, carried out simultaneously with the transit fit (the transit shape is preserved in this process).

${ }^{\mathrm{b}}$ Raw magnitude values without application of the EPD procedure. This is only reported for the follow-up light curves.

(This table is available in its entirety in machine-readable and Virtual Observatory (VO) forms.)

December 07. We used one of the three telescopes installed at the SAAO on the night of 2013 November 23 and one of the telescopes at CTIO on the night of 2013 December 07. In both cases we used the SBIG STX-16803 $4 \mathrm{~K} \times 4 \mathrm{~K}$ imagers with which the telescopes were initially deployed. These imagers provided a $16^{\prime} \times 16^{\prime}$ FOV with a pixel scale of $0{ }^{\prime \prime} 23$. We used a Pan-STARSS $z_{S}$ filter for the first night, and a Sloan $i^{\prime}$ filter for the second night. Other details regarding the instrumentation can be found in Brown et al. (2013). Calibrated science 

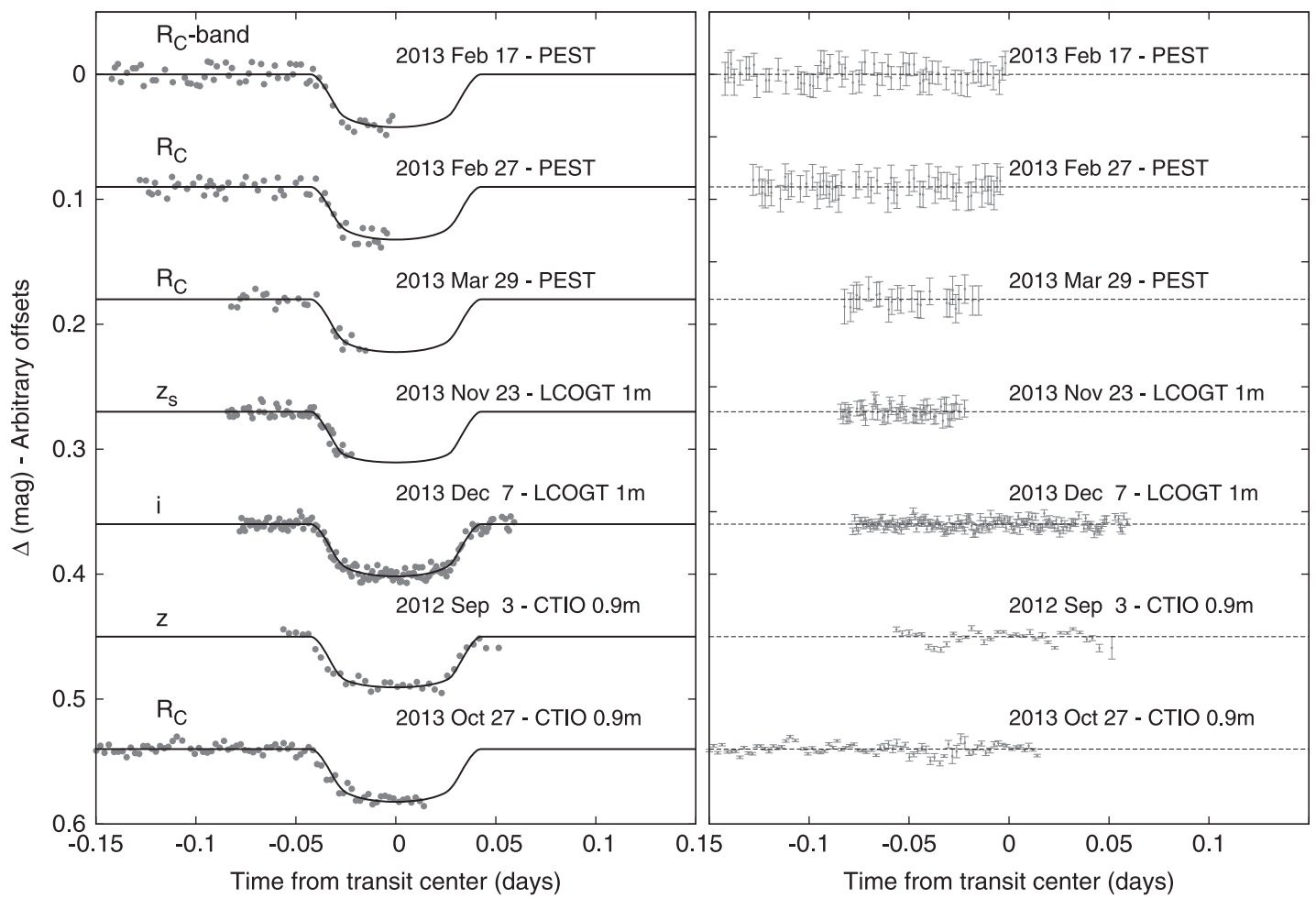

Figure 2. Left: unbinned follow-up transit light curves of HATS-6. The dates, filters and instruments used for each event are indicated. The light curves have been detrended using the EPD process. Curves after the first are shifted for clarity. Our best fit is shown by the solid lines. Right: residuals from the fits in the same order as the curves at left. Additional follow-up light curves from GROND are shown in Figure 3.
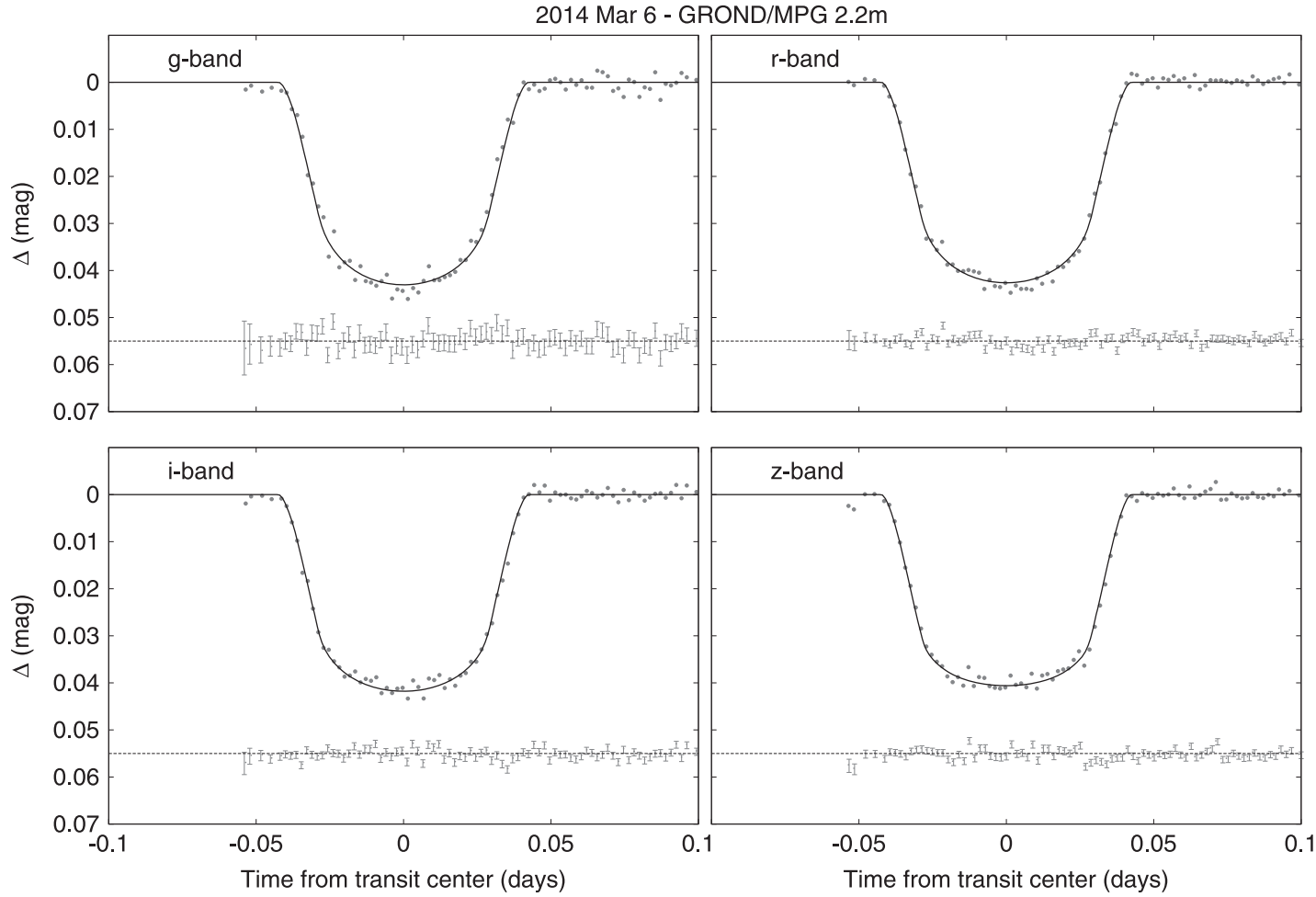

Figure 3. Unbinned instrumental Sloan $g-, r$-, $i$ - and $z$-band transit light curves of HATS-6 obtained with GROND on the MPG $2.2 \mathrm{~m}$ on the night of UT 2014 March 6. The light curves have been detrended using the EPD process, and the best-fit is shown by the solid lines. Below each light curve we plot the residuals.

images were provided by the LCOGT $1 \mathrm{~m}$ pipeline. We performed astrometry and aperture photometry using tools from the FITSH package (Pál 2012) on these images following methods that we have previously applied to observations from the Faulkes Telescopes, north and south, as well as to observations from Keplercam on the Fred Lawrence Whipple Observatory $1.2 \mathrm{~m}$ telescope. This procedure is described in Bakos et al. (2010). 
Table 3

Summary of Spectroscopic Observations

\begin{tabular}{llccc}
\hline \hline \multicolumn{1}{c}{ Telescope/Instrument } & \multicolumn{1}{c}{ Date Range } & Number of Observations & Resolution & Observing Mode \\
\hline ANU 2.3 m/WiFeS & 2012 May 13 & 1 & 3000 & Recon Spectral Type \\
ANU 2.3 m/WiFeS & 2012 Aug 6-Oct 24 & 4 & 7000 & Recon RVs \\
du Pont 2.5 m/Echelle & 2012 Oct 25-26 & 2 & 30000 & Recon RVs/Spectral Type \\
Magellan Clay 6.5 m/PFS & 2013 Nov 7-16 & 7 & 100000 & High Precision RVs \\
MPG 2.2 m/FEROS & 2013 Mar 24-May 11 & 8 & 48000 & High Precision RVs \\
ESO 3.6 m/HARPS & 2013 Dec 7-10 & 31 & 115000 & High Precision RVs \\
\hline
\end{tabular}

\subsection{Spectroscopy}

Table 3 summarizes the follow-up spectroscopic observations which we obtained for HATS-6.

\subsubsection{Reconnaissance Spectroscopy}

Initial reconnaissance spectroscopic observations of HATS-6 were carried out using the Wide Field Spectrograph (WiFeS; Dopita et al. 2007) on the ANU $2.3 \mathrm{~m}$ telescope at SSO together with the Echelle spectrograph on the du Pont $2.5 \mathrm{~m}$ telescope at LCO. The ANU $2.3 \mathrm{~m}$ data were reduced and analyzed following Bayliss et al. (2013), while for the du Pont data we used the pipeline we have previously developed (Jordán et al. 2014) to analyze data from the Coralie and FEROS spectrographs, adapted for the different spectral format of the instrument. A single WiFeS spectrum was obtained with a resolution of $R \equiv \lambda / \Delta \lambda=3000$ to use in measuring the effective temperature, surface gravity, and metallicity of the star, while four observations were obtained at a resolution of $R=7000$ to check for $\mathrm{RV}$ variations with amplitude $\gtrsim 5 \mathrm{~km} \mathrm{~s}^{-1}$ that would indicate that the transiting companion is of stellar mass. Based on the four WiFeS RV measurements, the $99 \%$ confidence upper limit on the orbital semiamplitude is $1.5 \mathrm{~km} \mathrm{~s}^{-1}$, which corresponds to an upper limit on the companion mass of $7.8 M_{J}$, assuming a $0.6 M_{\odot}$ primary star. The two du Pont spectra each had a resolution of $R=40,000$ covering a wavelength range of $3700-7000 \AA$, and were used to measure the effective temperature, gravity, metallicity, projected rotation velocity, and radial velocity of the star. Like the WiFeS spectra, the RV precision of the du Pont observations $\left(\sim 500 \mathrm{~m} \mathrm{~s}^{-1}\right)$ is not high enough to detect velocity variations due to a planet, but is sufficient to rule out stellarmass companions. The two du Pont RVs yield a $99 \%$ confidence upper limit of $1.4 \mathrm{~km} \mathrm{~s}^{-1}$ on the orbital semiamplitude, and an upper limit of $7.3 M_{J}$ on the mass of the companion.

Our analysis of the $R=3000 \mathrm{WiFeS}$ spectrum indicated an effective temperature of $T_{\text {eff } \star}=3600 \pm 300 \mathrm{~K}$, while the du Pont spectra yielded $T_{\text {eff } \star}=3700 \pm 100 \mathrm{~K}$. This effective temperature corresponds to a spectral type of M1 (Rajpurohit et al. 2013). The spectrum shows clear $\mathrm{TiO}$ absorption bands and is consistent with an M1V spectral classification. This spectrum also shows that HATS-6 is a quiet M dwarf with no evidence of emission in the $\mathrm{H} \alpha$ or $\mathrm{Ca}$ II $\mathrm{H}$ and $\mathrm{K}$ line cores. Additional indications that HATS-6 is a quiet star are the lack of any obvious starspot crossing events in the photometric follow-up light curves (Figures 2 and 3 ) and the lack of largeamplitude photometric variability in the HATSouth light curve. The WiFeS spectrum also indicated a dwarflike surface gravity $\left(\log g_{\star}=3.9 \pm 0.3\right.$; c.g.s. units) and a possibly sub-solar metallicity $([\mathrm{Fe} / \mathrm{H}]=-1.0 \pm 0.5)$, while our analysis of the du Pont spectra yielded a somewhat lower surface gravity $\left(\log g_{\star}=3.2 \pm 0.5\right)$ and metallicity $([\mathrm{Fe} / \mathrm{H}]=-1.5 \pm 0.5)$ and a moderately high rotation velocity $(v \sin i=7.5 \pm 2.0$ $\mathrm{km} \mathrm{s}^{-1}$ ). The analysis, however, relies on synthetic templates (MARCS models in the case of WiFeS and models from Coelho et al. 2005 in the case of du Pont) which are known to be unreliable for M-type stars. This means that systematic errors in the determined parameters are most likely greater than the estimated uncertainties (especially when $T_{\text {eff } \star}, \log g_{\star}$, and $[\mathrm{Fe} / \mathrm{H}]$, which are strongly correlated with each other, are all allowed to vary in fitting the spectra). As an example of this, note that based on the Dartmouth single stellar evolution models (Dotter et al. 2008) the minimum surface gravity realized for a $T_{\text {eff } \star}=3700 \mathrm{~K}$ dwarf star within $13.8 \mathrm{Gyr}$ is $\log g_{\star}=4.73$ which is significantly higher than the values determined from the spectroscopic modeling (the only evolved single stars that reach $T_{\text {eff } \star}=3700 \mathrm{~K}$ have $\log g_{\star} \lesssim 2$ ). We therefore do not consider the $\log g_{\star}[\mathrm{Fe} / \mathrm{H}]$, or $v \sin i$ measurements from this analysis to be reliable. More reliable estimates of $T_{\text {eff } \star}$ and $[\mathrm{Fe} / \mathrm{H}]$ are presented in the next section.

\subsubsection{Confirmation Spectroscopy}

In order to confirm HATS-6 as a transiting planet system through a detection of the RV orbital variation we obtained high-resolution spectra with three facilities capable of achieving $\sim 10 \mathrm{~m} \mathrm{~s}^{-1}$ or better RV precision. These are the FEROS spectrograph (Kaufer \& Pasquini 1998) on the MPG $2.2 \mathrm{~m}$ telescope at La Silla Observatory (LSO), the Planet Finder Spectrograph (PFS; Crane et al. 2010) on the Magellan Clay $6.5 \mathrm{~m}$ telescope at LCO, and the High Accuracy Radial Velocity Planet Searcher (HARPS; Mayor et al. 2003) spectrograph on the ESO $3.6 \mathrm{~m}$ telescope at LSO. Figure 4 shows the phased RV measurements and bisector spans from these observations, together with our best-fit orbit, while Table 4 lists the individual measurements.

A total of $8 R=48,000$ spectra were obtained with FEROS between 2013 March 24 and May 13. Three of these spectra suffered from very heavy sky contamination and have been excluded from our analysis (because RVs measured from these spectra are very unreliable we do not list these in Table 4 or show them in Figure 4). The other five exposures were also affected by sky contamination, however, in these cases the sky and target components were cleanly separated in the crosscorrelation functions (CCFs). Details on the FEROS spectra as used by HATSouth have been provided by Mohler-Fischer et al. (2013). For the observations reported we did not follow the reduction procedure described previously and instead reduced the data using an adapted version of the pipeline described by Jordán et al. (2014). This pipeline, which utilizes cross-correlation against binary templates, was originally developed for the CORALIE spectrograph on the Euler $1.2 \mathrm{~m}$ telescope at LSO. We found that applying this pipeline to 


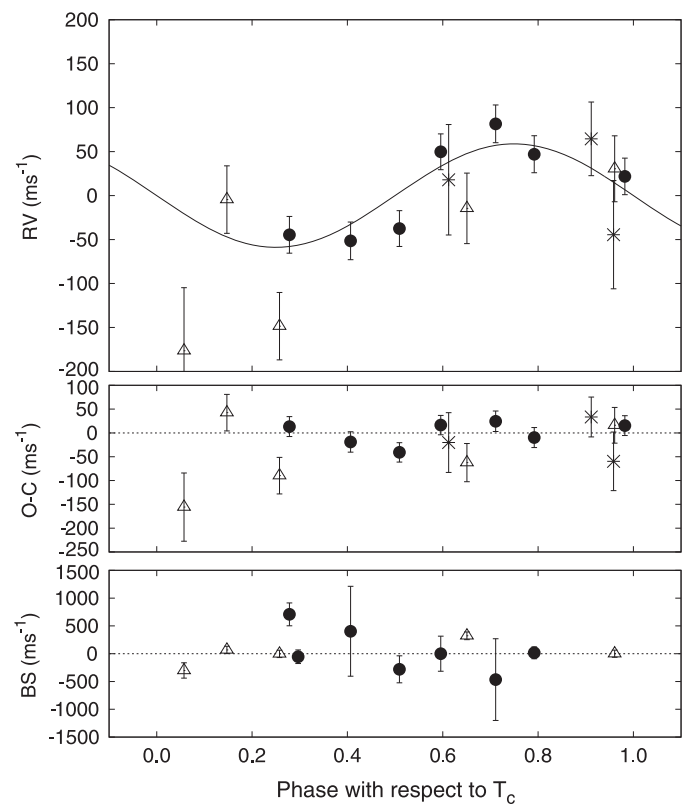

Figure 4. Top panel: high-precision RV measurements for HATS-6 from Magellan/PFS (dark filled circles), MPG $2.2 \mathrm{~m} /$ FEROS (open triangles), and ESO $3.6 \mathrm{~m} /$ HARPS (stars), together with our best-fit circular orbit model. Zero phase corresponds to the time of mid-transit. The center-of-mass velocity has been subtracted. The orbital fit is primarily constrained by the PFS observations. Second panel: velocity $O-C$ residuals from the best-fit model. The error bars for each instrument include the jitter which is varied in the fit. Third panel: bisector spans (BS), with the mean value subtracted. Note the different vertical scales of the panels.

FEROS data yields a precision of $\sim 8 \mathrm{~m} \mathrm{~s}^{-1}$ for RV standard stars, which is significantly better than the $\gtrsim 20 \mathrm{~m} \mathrm{~s}^{-1}$ precision previously achieved for this instrument. This will be described in additional detail in a separate paper (Brahm et al. in preparation). Bisector spans were also measured from the cross-correlation peak for the FEROS observations using this same pipeline, and following the method of Queloz et al. (2001).

The PFS observations consisted of an $\mathrm{I}_{2}$-free template spectrum obtained on the night of UT 2013 Nov 8, and seven observations taken through an $\mathrm{I}_{2}$ cell obtained between 2013 Nov 7 and 16. Observations were carried out with a $0.15 \times 2$ "! 5 slit, using $2 \times 2$ binning and slow read-out mode. Each observation with the $I_{2}$ cell in was split into three 20 minute exposures, while the $I_{2}$-free template was split into five 20 minute exposures. All but the last two observations were taken with the moon below the horizon. The combined spectra were reduced to RVs in the barycentric frame of the solar system following the method of Butler et al. (1996). We computed bisector spans in a manner similar to that used by Torres et al. (2007) to calculate bisector spans for Keck/HIRES data. In doing this we perform an order-by-order crosscorrelation between the observed spectra and a synthetic template. These are computed for the individual (non-stacked) exposures. The bisector spans are then measured from the resulting cross-correlation functions. We calculate the median bisector span for each order and subtract it from the measured spans. For a given observation we take the mean over the orders and individual exposures, taking the uncertainty to be the standard error on the mean. The reported bisector spans have had the median value subtracted. Note that the presence of the $\mathrm{I}_{2}$ cell restricts the spectral range over which the bisector spans may be computed. Whereas in Torres et al. (2007) and previous studies using Keck/HIRES data the bisector span analysis was done on the bluest spectral orders, which are free of $\mathrm{I}_{2}$ absorption, the faintness and extreme red color of HATS- 6 makes the signal blueward of $5000 \AA$ too low to be used for a bisector analysis. We therefore use five orders covering the

Table 4

Relative Radial Velocities and Bisector Span Measurements of HATS-6

\begin{tabular}{|c|c|c|c|c|c|c|}
\hline $\begin{array}{l}\text { BJD } \\
(2456000+)\end{array}$ & $\begin{array}{c}\mathrm{RV}^{\mathrm{a}} \\
\left(\mathrm{m} \mathrm{s}^{-1}\right)\end{array}$ & $\begin{array}{c}\sigma_{\mathrm{RV}}{ }^{\mathrm{b}} \\
\left(\mathrm{m} \mathrm{s}^{-1}\right)\end{array}$ & $\begin{array}{c}\mathrm{BS}^{\mathrm{c}} \\
\left(\mathrm{m} \mathrm{s}^{-1}\right)\end{array}$ & $\begin{array}{c}\sigma_{\mathrm{BS}} \\
\left(\mathrm{m} \mathrm{s}^{-1}\right)\end{array}$ & Phase & Instrument \\
\hline 377.58801 & 30.48 & 32.00 & 0.0 & 64.0 & 0.961 & FEROS \\
\hline 378.57524 & -148.52 & 33.00 & -3.0 & 66.0 & 0.258 & FEROS \\
\hline 401.48495 & -4.52 & 33.00 & 67.0 & 66.0 & 0.147 & FEROS \\
\hline 406.48479 & -14.52 & 35.00 & 322.0 & 70.0 & 0.651 & FEROS \\
\hline 424.46313 & -176.52 & 69.00 & -302.0 & 138.0 & 0.057 & FEROS \\
\hline $603.77801^{\mathrm{d}}$ & 21.86 & 7.09 & $\ldots$ & $\ldots$ & 0.982 & PFS \\
\hline 604.76307 & -44.62 & 7.36 & 708.1 & 205.7 & 0.278 & PFS \\
\hline $604.82373^{\mathrm{e}}$ & $\ldots$ & $\ldots$ & -53.9 & 122.4 & 0.297 & PFS \\
\hline 605.81892 & 49.82 & 5.79 & 0.0 & 315.3 & 0.596 & PFS \\
\hline 608.85541 & -37.49 & 5.80 & -280.7 & 243.2 & 0.509 & PFS \\
\hline 609.79629 & 47.03 & 7.77 & 15.7 & 107.1 & 0.792 & PFS \\
\hline $611.84038^{f}$ & -51.46 & 8.74 & 403.9 & 808.0 & 0.407 & PFS \\
\hline $612.85205^{\mathrm{f}}$ & 81.60 & 8.84 & -465.6 & 735.3 & 0.711 & PFS \\
\hline 633.62669 & -44.47 & 58.38 & $\ldots$ & $\ldots$ & 0.958 & HARPS \\
\hline 635.80096 & 18.01 & 59.69 & $\ldots$ & $\ldots$ & 0.612 & HARPS \\
\hline 636.79588 & 64.54 & 37.05 & $\ldots$ & $\ldots$ & 0.912 & HARPS \\
\hline
\end{tabular}

\footnotetext{
${ }^{a}$ The zero-point of these velocities is arbitrary. An overall offset $\gamma_{\text {rel }}$ fitted separately to the PFS, HARPS, and FEROS velocities in Section 3 has been subtracted.

${ }^{\mathrm{b}}$ Internal errors excluding the component of astrophysical/instrumental jitter considered in Section 3.

${ }^{\mathrm{c}}$ The median BS value has been subtracted for each instrument.

${ }^{d}$ The CCF peak height was too low in the orders where we computed the BS to be able to extract a BS measurement for this observation.

e This PFS observation was taken without the iodine cell to be used as a template. The RV is not measured for this observations, but BS value is measured.

${ }^{\mathrm{f}}$ These PFS observations have high sky contamination relative to the other PFS observations which were obtained with the moon below the horizon. The $\sigma_{\mathrm{BS}}$ values for these two exposures are large as a result.
} 
spectral range 6200-6540 $\AA$. The limited spectral range reduces the precision of the bisector spans. In fact we find that the bisector spans calculated from the MPG $2.2 \mathrm{~m} / \mathrm{FEROS}$ data have lower scatter than those from Magellan/PFS due to the greater spectral range used for the FEROS measurements.

We used our $\mathrm{I}_{2}$-free template spectrum from PFS to measure $T_{\text {eff }}$ and $[\mathrm{Fe} / \mathrm{H}]$ for HATS- 6 following the method of Neves et al. (2014). We made use of the Python routines referenced in that paper to perform this analysis after modifying them for the spectral range of PFS (we verified that the code reproduces the temperature and metallicity of the two HARPS spectra supplied with the routines when applied to the restricted spectral range). We find $T_{\text {eff } \star}=3770 \pm 100 \mathrm{~K}$ and $[\mathrm{Fe} / \mathrm{H}]=0.200 \pm 0.091$, where the errors are determined from Table 6 of Neves et al. (2014) scaling by $\sqrt{N_{\text {all }} / N_{\text {used }}}$ where $N_{\text {all }}$ is the number of spectral lines used by Neves et al. (2014), and $N_{\text {used }}$ is the number of lines within the PFS spectral range.

The HARPS observations consisted of three exposures taken on the nights of UT 2013 December 7-9. We used an exposure time of $1200 \mathrm{~s}$ on the first night and $1800 \mathrm{~s}$ on the following two nights. Observations were carried out in the "object+sky" mode (due to the faintness of the target, contamination from scattered moonlight is substantial for the first exposure; the second and third HARPS observations were made with the moon below the horizon) and reduced to RVs using the facility data reduction software together with a K star spectral mask (at present this is the coolest facility mask available). The spectra have a resolution of $R=115,000$ covering a range of $378-691 \mathrm{~nm}$.

In practice, due to the faintness of HATS- 6 and the low mass of the planet HATS-6b, the orbital variation is detected with significance only from the PFS data. We include the data from HARPS and FEROS in our RV model (Section 3.2) for completeness, and to ensure that the fit accounts for all data, including non-detections.

\section{ANALYSIS}

\subsection{Excluding Blend Scenarios}

In order to rule out the possibility that HATS-6 is not a planetary system, but is instead a blend between an eclipsing binary star and another source, we carried out a blend analysis similar to that done in Hartman et al. (2012), with a difference being that in this case we use the Dartmouth (Dotter et al. 2008) stellar evolution models to calculate the properties of simulated blended systems. We find that although blend models exist involving an eclipsing binary blended with a brighter foreground $\mathrm{M}$ dwarf, which match the light curves and absolute photometry, in all such cases both the foreground source and the primary in the background binary have an apparent magnitude difference $|\Delta V|<1 \mathrm{mag}$, so that crosscorrelation functions (CCFs) computed from the PFS, FEROS, and HARPS spectra would show obvious secondary peaks and $\mathrm{RV}$ variations greater than $1 \mathrm{~km} \mathrm{~s}^{-1}$. The simulated CCFs are grossly inconsistent with the observed CCFs, so we conclude that this is not a blended eclipsing binary system and is instead a transiting planet system. We note that there is no significant trend in the observed bisector spans from FEROS or PFS nor is there any evidence for more than one stellar component in the cross-correlation functions.
As is often the case we cannot exclude the possibility that HATS-6 is an unresolved binary system with one component having a transiting planet. High resolution imaging would provide constraints on any such wide binary companions. For the analysis presented here we assume that this is an isolated star. If future observations reveal that this is a binary system, corrections to the planet mass and radius would increase their values from those presented in this paper.

\subsection{Global Fit of Light Curves and RV Measurements}

In order to determine the physical parameters of the HATS-6 system we carried out an analysis similar to that described in Bakos et al. (2010) and Penev et al. (2013). All light curves (HATSouth data and follow-up data) and RV measurements are simultaneously fitted using a Mandel \& Agol (2002) transit light curve model and a Keplerian RV orbit.

The light curve model is extended using a model for instrumental variations such that the total model can be expressed as:

$$
\begin{aligned}
m_{k, i}= & m_{k, 0}+\Delta m\left(t_{i} ; T_{0}, T_{N_{t}}, \zeta / R_{\star}, R_{p} / R_{\star}, b, a_{\mathrm{LD}, k}, b_{\mathrm{LD}, k}\right) \\
& +\sum_{j=1}^{N_{\mathrm{EPD}, k}} c_{\mathrm{EPD}, k, j} x_{k, j, i}+\sum_{j=1}^{N_{\mathrm{TFA}}} c_{\mathrm{TFA}, j} y_{k, j, i}
\end{aligned}
$$

where $m_{k, i}$ is the measured magnitude to be modeled for observation $i$ of light curve $k ; m_{k, 0}$ is the zero-point magnitude for light curve $k$ (which is a free parameter in the model); $\Delta m\left(t_{i} ; T_{0}, T_{N_{t}}, \zeta / R_{\star}, R_{p} / R_{\star}, b, a_{\mathrm{LD}, k}, b_{\mathrm{LD}, k}\right)$ is the physical Mandel \& Agol (2002) model evaluated at time $t_{i}$ and parameterized by initial and final transit epochs $T_{0}$ and $T_{N_{t}}$ (the period is then given by $\left.\left(T_{N_{t}}-T_{0}\right) / N_{t}\right)$, reciprocal of the half duration of the transit $\zeta / R_{\star}$, ratio of the planetary and stellar radii $R_{p} / R_{\star}$, normalized impact parameter $b$, and quadratic limb darkening coefficients $a_{\mathrm{LD}, k}$ and $b_{\mathrm{LD}, k}$ appropriate for the filter of light curve $k$ (except for $a_{\mathrm{LD}, k}$ and $b_{\mathrm{LD}, k}$, which are fixed using the tabulations of Claret 2004, these parameters are varied in the fit). There are $N_{\mathrm{EPD}, k}$ EPD parameters applied to light curve $k$ with $c_{\mathrm{EPD}, k, j}$ being the free coefficient fitted for EPD parameter series $j$ applied to light curve $k$, and $x_{k, j, i}$ being the value of EPD parameter series $j$ at observation $i$ for light curve $k$ and there are $N_{\text {TFA }}$ TFA templates used to fit the light curve, with $c_{\mathrm{TFA}, j}$ being the free coefficient fitted for template $j$ and $y_{k, j, i}$ being the value of template $j$ at observation $i$ for light curve $k$. For the HATSouth light curve we do not include the EPD and TFA terms in the fit, and instead model the light curve that was pre-processed through these filtering routines without accounting for the transits. In this case we also include an instrumental blending factor (varied in the fit) which scales the depth of the Mandel \& Agol (2002) model applied to the HATSouth light curve by a factor between 0 and 1 (assuming a uniform prior between these limits) to account for both blending from nearby stars as well as the artificial dilution of signals due to the filtering.

For the RV model we allow an independent RV zero-point, and an independent RV jitter for each of the three instruments used. The jitter is a term added in quadrature to the formal RV 
uncertainties for each instrument and is varied in the fit following Hartman et al. (2012).

We use a Differential Evolution Markov Chain Monte Carlo (DEMCMC) procedure (ter Braak 2006; Eastman et al. 2013) to explore the fitness of the model over parameter space and produce a chain of parameters drawn from the posterior distribution. This chain is then used to estimate the most likely value (taken as the median value over the chain) together with the $68.3 \%(1 \sigma)$ confidence interval for each of the physical parameters.

The fit is performed both allowing the eccentricity to vary and fixing it to zero. We find that without additional constraints, the free-eccentricity model strongly prefers a non-zero eccentricity of $e=0.404 \pm 0.058$. This is entirely due to the PFS velocities which closely follow such an eccentric orbit with a near-zero jitter for the PFS RVs of $0.1 \pm 5.6 \mathrm{~m} \mathrm{~s}^{-1}$. When the eccentricity is fixed to zero, on the other hand, the PFS RVs are consistent with a circular orbit, but in this case require a jitter of $26 \pm 14 \mathrm{~m} \mathrm{~s}^{-1}$. Due to the faintness of HATS- 6 in the optical band-pass, and consequent sky contamination, such a high "jitter" is not unreasonable and may simply reflect an underestimation of the formal RV uncertainties. As we discuss below, the stellar parameters inferred for the high eccentricity solution are inconsistent with the spectroscopic parameters and broad-band photometric colors of the star. When the broad-band photometric color measurements are directly folded into our light curve and RV modeling through an empirical stellar model, as we discuss in Section 3.3.2, the preferred eccentricity is consistent with zero $(e=0.053 \pm 0.060$, and a $99 \%$ confidence upper limit of $e<0.25)$.

\subsection{Determining the Physical Parameters of the Star and Planet}

To determine the mass and radius of the transiting planet from the physical parameters measured above requires knowledge of the stellar mass and radius. For a non-binary star such as HATS-6 these parameters are not easy to measure directly and instead must be inferred by comparing other measurable parameters, such as the surface temperature and bulk stellar density, with theoretical stellar evolution models (requiring the metallicity, a color indicator, and a luminosity indicator to identify a unique stellar model), or with empirical relations calibrated using binary stars. We considered both methods, discussed in turn below.

\subsubsection{Dartmouth Models}

Because the star is a cool dwarf we make use of the Dartmouth stellar evolution models (Dotter et al. 2008) which appear to provide the best match to $\mathrm{M}$ dwarf and late $\mathrm{K}$ dwarf stars (e.g., Feiden et al. 2011; Sandquist et al. 2013). We also use the effective temperature and metallicity measured from the PFS $\mathrm{I}_{2}$-free template spectrum.

We use the results from our DEMCMC analysis of the light curve and radial velocity data (Section 3.2) together with the Dartmouth isochrones to determine the stellar parameters. For each density measurement in the posterior parameter chain we associate $T_{\text {eff }}$ and $[\mathrm{Fe} / \mathrm{H}]$ measurements drawn from Gaussian distributions. We look up a matching stellar model from the Dartmouth isochrones, interpolating between the tabulated models, and append the set of stellar parameters associated with this model to the corresponding link in the posterior parameter chain. Other planetary parameters, such as the mass and radius, which depend on the stellars parameters, are then calculated for each link in the chain.

Figure 5 compares the measured $T_{\text {eff }}$ and $\rho_{\star}$ values for HATS-6 $\left(\rho_{\star}=4.40 \pm 0.13 \mathrm{~g} \mathrm{~cm}^{-3}\right.$, and $T_{\text {eff } \star}=3770 \pm 100$ $\mathrm{K})$ to the interpolated $[\mathrm{Fe} / \mathrm{H}]=0.20$ Dartmouth isochrones. We also compare the $V-K$ and $\rho_{\star}$ values to these same isochrones. For reference we show seven transiting-planethosting stars with $T_{\mathrm{eff} \star}, V-K, \rho_{\star}$ and $M_{\star}$ similar to that of HATS-6. These are listed in Table 8 as well. At fixed $T_{\text {eff }}$ or $V-K$ the models predict $\rho_{\star}$ values that are slightly lower than those seen among the transit hosts. This suggests that using $V-K$ as an input in constraining the mass of the star through the Dartmouth models may lead to a slightly overestimated stellar mass (lower densities yield higher masses). For comparison we also show the relations derived from the empirical relations discussed below. The empirical relations appear to provide a better match to the stars shown in Figure 5.

As noted in Section 3.2 when we allow the eccentricity to vary in the fit the PFS data pull the model toward a high eccentricity solution $(e=0.404 \pm 0.058)$ which also yields a high stellar density of $14.8 \pm 2.9 \mathrm{~g} \mathrm{~cm}^{-3}$. The combination of the high stellar density, hot effective temperature $\left(T_{\text {eff } \star}=3770 \pm 100 \mathrm{~K}\right)$, and high metallicity $([\mathrm{Fe} / \mathrm{H}]=$ $0.200 \pm 0.091)$ cannot be matched by the Dartmouth isochrones. If we instead use $V-K$ as the temperature indicator and draw metallicities from the Haywood (2001) solar neighborhood metallicity distribution we find that only very low metallicity models $([\mathrm{Fe} / \mathrm{H}]=-0.63 \pm 0.07)$ match the observations. Such a metallicity is at odds with the spectroscopic measurement. Moreover, taking the parameters from the eccentric model and assuming $Q_{P}=10^{6}$ the circularization timescale (e.g., Adams \& Laughlin 2006) is only $342 \pm$ $35 \mathrm{Myr}$. No planets with $t_{\text {circ }}<1 \mathrm{Gyr}$ have been found with eccentricities greater than 0.1 (e.g., see Figure 12 of Bakos et al. 2012), making it unlikely that HATS-6b has such a high eccentricity.

\subsubsection{Empirical Relations}

As an alternative method for determining the stellar parameters, and to better understand the degree of systematic errors in these parameters, we also develop a set of empirical relations between stellar density, which is directly measured for a transiting planet system, and other stellar parameters. Such relations have been developed and employed previously in transiting exoplanet studies (Enoch et al. 2010; Torres et al. 2010; Southworth 2011). The Torres et al. (2010) and Enoch et al. (2010) relations only considered stars with $M>0.6 M_{\odot}$, making them inapplicable in this case. The Southworth (2011) relations consider stars over the range $0.2 M_{\odot}<M<3.0 M_{\odot}$. They present two relations, one for mass as a function of temperature, density, and metallicity and the other for radius as a function of temperature, density, and metallicity. Fitting these as two independent functions ignores the fact that density, mass, and radius must satisfy the relation $M=\frac{4}{3} \pi R^{3} \rho$. Moreover, one should not expect the scaling of mass and radius with metallicity or temperature to be independent of stellar mass over such a broad range in mass. And, since 

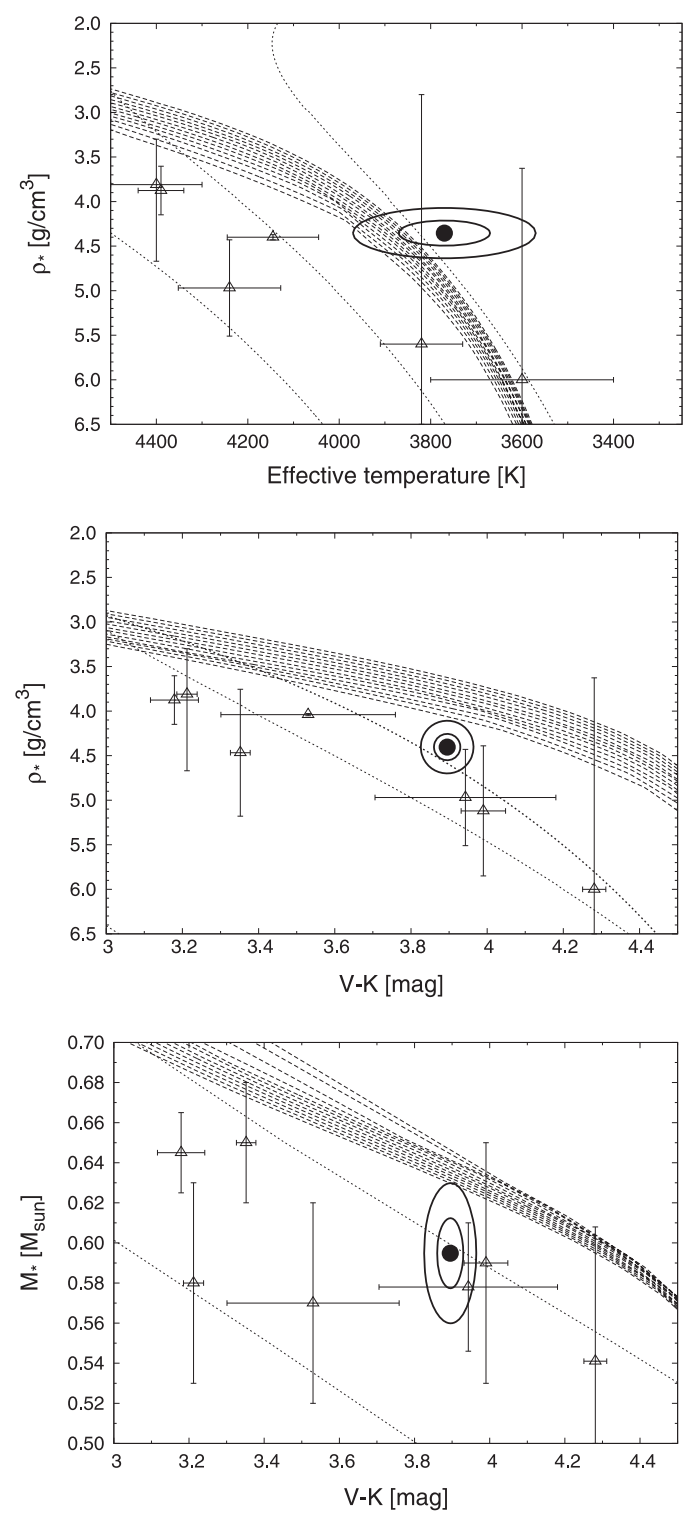

Figure 5. Model isochrones (dashed lines) from Dotter et al. (2008) for the spectroscopically determined metallicity of HATS-6 and ages of 1-13 Gyr in 1 Gyr steps (showing $\rho_{\star}$ vs. $T_{\text {eff }}$ at top, $\rho_{\star}$ vs. $V-K$ in the middle, and $M_{\star}$ vs. $V-K$ at the bottom). The measured values of $T_{\text {eff }}, V-K$, and $\rho_{\star}$ for HATS-6, and the value of $M_{\star}$ inferred from comparison to the stellar models, are shown using the large filled circles together with their $1 \sigma$ and $2 \sigma$ confidence ellipsoids. The open triangles show other transiting planet host stars with measured $T_{\text {eff }}$, $V-K, \rho_{\star}$, and $M_{\star}$ values similar to HATS-6. Near the K/M spectral type boundary $(V-K \sim 4)$ the models predict somewhat lower densities for a given $V-K$ or $T_{\text {eff }}$ than are seen among transit hosts (Table 8). For comparison we also show the relations derived from our empirical model (dotted lines; Section 3.3.2). In the top panel we show the three dotted lines are the median relation, and the $1 \sigma$ lower and upper bounds. In the bottom two panels the lower dotted line is the median relation, while the upper dotted line is the $1 \sigma$ upper bound. The $1 \sigma$ lower bound from the empirical model lies just outside the range of these plots (below the bottom left corner in each case). The empirical models appear to be more consistent with the transiting planet hosts than the theoretical models, but also cover a broader range of parameter space than seen among the transit hosts.

metallicity is available for only a very few $\mathrm{M}$ dwarf eclipsing binaries, the fit performed by Southworth (2011) effectively imposes the metallicity scaling for A through $\mathrm{G}$ stars on the $\mathrm{M}$ dwarfs. We therefore consider it worthwhile to revisit these relations for $\mathrm{K}$ and $\mathrm{M}$ dwarf stars.
Johnson et al. (2011) and Johnson et al. (2012) have also developed empirical relations for characterizing $\mathrm{M}$ dwarf planet hosts, applying them to the characterization of LHS $6343 \mathrm{AB}$ and Kepler-45, respectively. Our approach is similar to theirs in that we make use of relations between mass and absolute magnitudes based on data from Delfosse et al. (2000), and we also consider the empirical mass-radius relation based on eclipsing binaries, however, we differ in the sample of eclipsing binaries that we consider, and we adopt a different parameterization of the problem. Moreover, while Johnson et al. (2012) use several empirical relations which were independently fit using different data sets, our approach is to self-consistently determine all of the relations through a joint analysis of the available data. We discuss this comparison in more detail below.

We look for the following relations: (1) $\rho_{\star} \rightarrow R_{\star}$ (which also defines a $\rho_{\star} \rightarrow M_{\star}$ relation), (2) $M_{\star} \rightarrow M_{\mathrm{bol}}$, (which together with relation 1 also defines a relation between $T_{\text {eff } \star}$ and the other parameters) (3) $T_{\text {eff } \star} \rightarrow M_{\mathrm{bol}}-M_{V}$, (4) $T_{\text {eff } \star} \rightarrow$ $M_{\mathrm{bol}}-M_{J}$, (5) $T_{\mathrm{eff} \star} \rightarrow M_{\mathrm{bol}}-M_{H}$, (6) $T_{\mathrm{eff} \star} \rightarrow M_{\mathrm{bol}}-M_{K}$. The motivation for choosing this particular formulation is that $\rho_{\star}$ is typically a well-measured parameter for transiting planet systems, and the relation between $\rho_{\star}$ and $R_{\star}$ is tighter than for other relations involving $\rho_{\star}$. The other relations are then based on physical dependencies (bolometric magnitude depends primarily on stellar mass, with age and metallicity being secondary factors, and bolometric corrections depend primarily on effective temperature, with metallicity being a secondary factor). These relations are parameterized as follows:

$$
\begin{aligned}
& l R=\left(\begin{array}{c}
a_{\rho, 0} \\
a_{\rho, 1} \\
a_{\rho, 2} \\
a_{\rho, 3}
\end{array}\right)^{T} \\
& \left(\begin{array}{cccc}
0.131674 & -0.605624 & 0.739127 & -0.263765 \\
-0.494952 & 0.614479 & 0.437886 & -0.430922 \\
-0.765425 & -0.291096 & 0.099548 & 0.565224 \\
0.389626 & 0.413398 & 0.502032 & 0.652117
\end{array}\right) \\
& \left(\begin{array}{c}
1 \\
l \rho \\
l \rho^{2} \\
l \rho^{3}
\end{array}\right)+d l R \\
& M_{\mathrm{bol}}=\left(\begin{array}{c}
b_{m, 0} \\
b_{m, 1} \\
b_{m, 2} \\
b_{m, 3}
\end{array}\right)^{T} \\
& \left(\begin{array}{cccc}
-0.042114 & -0.349391 & -0.787638 & -0.505746 \\
0.170316 & 0.723492 & 0.095153 & -0.662192 \\
-0.309788 & -0.505183 & 0.591641 & -0.546610 \\
-0.934479 & 0.315080 & -0.143296 & 0.083309
\end{array}\right) \\
& \left(\begin{array}{c}
1 \\
l M \\
l M^{2} \\
l M^{3}
\end{array}\right)+d M_{\mathrm{bol}}
\end{aligned}
$$




$$
\begin{gathered}
B C_{\lambda}=\left(\begin{array}{l}
c_{\lambda, 0} \\
c_{\lambda, 1} \\
c_{\lambda, 2}
\end{array}\right)^{T} \\
\left(\begin{array}{ccc}
0.871464 & -0.485768 & 0.067675 \\
0.484681 & 0.831841 & -0.270418 \\
0.075065 & 0.268461 & 0.960361
\end{array}\right) \\
\left(\begin{array}{c}
1 \\
l T \\
l T^{2}
\end{array}\right)
\end{gathered}
$$

where $l R=\log _{10}(R), l \rho=\log _{10}(\rho), l M=\log _{10}(M)$, and $l T=$ $\log _{10}\left(T_{\text {eff }}\right)$ are the logarithms of the stellar radius in solar units, density in cgs units, mass in solar units and temperature in Kelvin, respectively. The sets of coefficients in the vectors to the left of the matrices (e.g., $a_{\rho, 0}, a_{\rho, 1}$; optimal values are given in Table 7) are varied in the fit, and the matrices are chosen to minimize correlations between these parameters. The matrices are determined by initially fitting the relations using simple polynomials (i.e., we replace the matrices in the relations above with the identity matrix) and then performing a principal component analysis on the resulting Markov Chains to determine the transformation to a linearly uncorrelated set of parameters. These matrices are held fixed in a subsequent fit where we determine the optimized values of the parameters $a_{\rho, 0}, a_{\rho, 1}$, etc. (see below for more discussion of the fitting procedure). The term $\lambda$ is either $V, J, H$, or $K$. To allow for intrinsic scatter in the relations, the terms $d l R$ and $d M_{\mathrm{bol}}$ represent real deviations in the radius or bolometric magnitude from the model. Such deviations are expected due to additional parameters, such as the metallicity or age, which are not included in this model. Every star has its own value of $d l R$ and $d M_{\text {bol }}$, assumed to be drawn from Gaussian distributions with standard deviations of $S_{R}$ and $S_{\text {Mbol }}$, respectively. The following auxiliary relations are also used to relate the stellar density to the mass, the bolometric magnitude and radius to the effective temperature, and the bolometric magnitude and bolometric correction to the absolute magnitude in a given filter $\left(M_{\lambda}\right)$ :

$$
\begin{gathered}
l M=l \rho-0.14968+3 l R(l \rho) \\
l T=\left(42.227-5 l R-M_{\mathrm{bol}}\right) / 10 \\
M_{\lambda}=M_{\mathrm{bol}}+B C_{\lambda} .
\end{gathered}
$$

The stars that we use in the fit are listed in Tables 5 and 6. The eclipsing binaries are compiled from tables in Zhou et al. (2014), Torres et al. (2010), and Nefs et al. (2013), and include non-pre-main-sequence stars with $M_{\star}<0.85 M_{\odot}$ and with $M_{\star}$ and $R_{\star}$ measured to better than $5 \%$ accuracy. The upper mass limit corresponds roughly to the mass for which stars older than the pre-main-sequence phase and with $-0.5<[\mathrm{Fe} / \mathrm{H}]<0.5$ will have $\log g>4.4$ throughout the $\sim 10 \mathrm{Gyr}$ age of the Galactic disk. Stars below this mass show tight correlations between the density and the mass, radius, and luminosity. Because individual broad-band photometric magnitudes are not available for most of the eclipsing binary components, we use resolved binaries for which masses and component magnitudes are available to constrain relations (3) through (6) above. Most of these stars are taken from the compilation by Delfosse et al. (2000), with a few objects from the list of Torres et al. (2010) to extend into the late $\mathrm{K}$ dwarf regime.

To fit the above relations we use a likelihood function of the form:

$$
\begin{aligned}
\ln L= & -\frac{1}{2} \sum_{i=1}^{N_{\star}}\left(\frac{l R\left(l \rho_{i}\right)-l R_{\mathrm{obs}, i}}{\sigma_{l R, i}}\right)^{2} \\
& -\frac{1}{2} \sum_{i=1}^{N_{\star}}\left(\frac{l M\left(l \rho_{i}\right)-l M_{\mathrm{obs}, i}}{\sigma_{l M, i}}\right)^{2} \\
& -\frac{1}{2} \sum_{i=1}^{N_{\star}}\left(\frac{\left.l T\left(l R\left(l \rho_{i}\right), M_{\mathrm{bol}}\left(l M\left(l \rho_{i}\right)\right)\right)-l T_{\mathrm{obs}, i}\right)^{2}}{\sigma_{l T, i}}\right)^{2} \\
& -\frac{1}{2} \sum_{\lambda} \sum_{i=1}^{N_{\star}}\left(\left(M_{\mathrm{bol}}\left(l M\left(l \rho_{i}\right)\right)+B C_{\lambda}\left(l T \left(l R\left(l \rho_{i}\right)\right.\right.\right.\right. \\
& \left.\left.\left.-\frac{1}{2} \sum_{i=1}^{N_{\star}}\left(l M\left(l \rho_{i}\right)\right)\right)-M_{\lambda, \mathrm{obs}, i}\right) / \sigma_{\lambda, i}\right)^{2}\left(\ln \left(S_{R}^{2}\right)+\left(\frac{d l R_{i}}{S_{R}}\right)^{2}\right) \\
& -\frac{1}{2} \sum_{i=1}^{N_{\star}}\left(\ln \left(S_{\mathrm{Mbol}}^{2}\right)+\left(\frac{d M_{\mathrm{bol}, i}}{S_{\mathrm{Mbol}}}\right)^{2}\right)
\end{aligned}
$$

where the sum on $\lambda$ is over the four filters $V, J, H$, and $K$, and for each star we exclude terms from the sums if the relevant measurement $\left(l R_{\mathrm{obs}}, l M_{\mathrm{obs}}, l T_{\mathrm{obs}}, M_{V, \mathrm{obs}}, M_{J, \mathrm{obs}}, M_{H, \mathrm{obs}}\right.$, or $\left.M_{K, \mathrm{obs}}\right)$ is not available. Here $l \rho_{i}, d l R_{i}$, and $d M_{\mathrm{bol}, i}$ are free parameters for each star $i$, and the other free parameters are $S_{R}$, $S_{\mathrm{Mbol}}$, and the coefficients in Equations (2)-(4). We use the DEMCMC procedure to explore this likelihood function. Table 7 provides the values for the best-fit model together with their approximate $1 \sigma$ (uncorrelated) uncertainties. ${ }^{13}$

Figure 6 shows the fit between $l \rho, l M$, and $l R$, and between $I M$ and $M_{\mathrm{bol}}$, together with the residuals from this model; Figure 7 shows the relations between effective temperature and the $V, J, H$, and $K$ bolometric corrections; Figure 8 shows the relation between mass and the absolute magnitudes in each of these filters; and Figure 9 shows the residuals from the fits in Figure 8 . Our model yields intrinsic scatters of $\sim 2 \%$ in radius and $\sim 7 \%$ in mass, and $4 \%-10 \%$ in effective temperature (between 0.2 and $0.5 \mathrm{mag}$ in bolometric magnitudes) given a value for $\rho_{\star}$. Figure 10 compares the precision in the inferred mass and radius implied by this modeling when using a single parameter to constrain the stellar properties (either $\rho_{\star}, M_{V}, M_{K}$, or $V-K)$, and when using various combinations of observables. Assuming no observational uncertainties, $M_{K}$ is the highest precision stellar mass indicator, while $\rho_{\star}$ is the highest precision stellar radius indicator. By combining $\rho_{\star}$ with a photometric indicator, such as $M_{K}$, it is possible to improve the precision in mass and radius by up to a factor of two compared to the precision allowed by using only $\rho_{\star}$.

\footnotetext{
13 A C code implementing these relations to determine the properties of $\mathrm{K}$ and $\mathrm{M}$ dwarf stars from various observed quantities is available at http://www.astro. princeton.edu/jhartman/kmdwarfparam.html.
} 
Table 5

K and M Dwarf Binary Components Used to Fit Empirical Relations ${ }^{\mathrm{a}}$

\begin{tabular}{|c|c|c|c|c|}
\hline Star & $\begin{array}{l}\text { Mass } \\
\left(M_{\odot}\right)\end{array}$ & $\begin{array}{c}\text { Radius } \\
\left(R_{\odot}\right) \\
\end{array}$ & $\begin{array}{l}T_{\text {eff }} \\
(\mathrm{K})\end{array}$ & Reference \\
\hline 1 RXS J154727.5+450803 A & $0.2576 \pm 0.0085$ & $0.2895 \pm 0.0068$ & $\ldots$ & 1 \\
\hline 1 RXS J154727.5+450803 B & $0.2585 \pm 0.0080$ & $0.2895 \pm 0.0068$ & $\ldots$ & 1 \\
\hline ASA J011328-3821.1 A & $0.612 \pm 0.03$ & $0.596 \pm 0.02$ & $3750 \pm 250$ & 2 \\
\hline CG Cyg B & $0.814 \pm 0.013$ & $0.838 \pm 0.011$ & $4720 \pm 60$ & 3,4 \\
\hline CM Dra A & $0.2130 \pm 0.0009$ & $0.2534 \pm 0.0019$ & $3130 \pm 70$ & 5 \\
\hline CM Dra B & $0.2141 \pm 0.0010$ & $0.2396 \pm 0.0015$ & $3120 \pm 70$ & 5 \\
\hline GJ $3236 \mathrm{~A}$ & $0.376 \pm 0.016$ & $0.3795 \pm 0.0084$ & $3310 \pm 110$ & 7 \\
\hline GJ $551^{\mathrm{b}}$ & $0.123 \pm 0.006$ & $0.141 \pm 0.007$ & $3098 \pm 56$ & $8,9,10$ \\
\hline GU Boo A & $0.610 \pm 0.006$ & $0.627 \pm 0.016$ & $3920 \pm 130$ & 11 \\
\hline GU Boo B & $0.600 \pm 0.006$ & $0.624 \pm 0.016$ & $3810 \pm 130$ & 11 \\
\hline HATS550-016 & $0.110_{-0.006}^{+0.005}$ & $0.147_{-0.004}^{+0.003}$ & $\ldots$ & 12 \\
\hline HATS551-021 & $0.132_{-0.005}^{+0.014}$ & $0.154_{-0.008}^{+0.006}$ & $\ldots$ & 12 \\
\hline KOI-126 B & $0.2413 \pm 0.0030$ & $0.2543 \pm 0.0014$ & $\ldots$ & 17 \\
\hline KOI-126 C & $0.2127 \pm 0.0026$ & $0.2318 \pm 0.0013$ & $\ldots$ & 17 \\
\hline LSPM J1112+7626 A & $0.3946 \pm 0.0023$ & $0.3860 \pm 0.0055$ & $3060 \pm 160$ & 18 \\
\hline LSPM J1112+7626 B & $0.2745 \pm 0.0012$ & $0.2978 \pm 0.0049$ & $2950 \pm 160$ & 18 \\
\hline MG1-116309 A & $0.567 \pm 0.002$ & $0.552 \pm 0.0085$ & $3920 \pm 80$ & 19 \\
\hline MG1-116309 B & $0.532 \pm 0.002$ & $0.532 \pm 0.006$ & $3810 \pm 80$ & 19 \\
\hline MG1-1819499 A & $0.557 \pm 0.001$ & $0.569 \pm 0.0022$ & $3690 \pm 80$ & 19 \\
\hline MG1-1819499 B & $0.535 \pm 0.001$ & $0.500 \pm 0.0085$ & $3610 \pm 80$ & 19 \\
\hline MG1-2056316 A & $0.4690 \pm 0.0021$ & $0.441 \pm 0.002$ & $3460 \pm 180$ & 19 \\
\hline MG1-2056316 B & $0.382 \pm 0.001$ & $0.374 \pm 0.002$ & $3320 \pm 180$ & 19 \\
\hline MG1-506664 A & $0.584 \pm 0.002$ & $0.560 \pm 0.0025$ & $3730 \pm 90$ & 19 \\
\hline MG1-506664 B & $0.544 \pm 0.002$ & $0.513 \pm 0.0055$ & $3610 \pm 90$ & 19 \\
\hline V568 Lyr B & $0.8273 \pm 0.0042$ & $0.7679 \pm 0.0064$ & $4900 \pm 100$ & 24 \\
\hline WOCS 23009 B & $0.447 \pm 0.011$ & $0.4292 \pm 0.0033$ & $3620 \pm 150$ & 25 \\
\hline WTS19b-2-01387 A & $0.498 \pm 0.019$ & $0.496 \pm 0.013$ & $3498 \pm 100$ & 26 \\
\hline WTS19b-2-01387 B & $0.481 \pm 0.017$ & $0.479 \pm 0.013$ & $3436 \pm 100$ & 26 \\
\hline YY Gem A & $0.599 \pm 0.005$ & $0.619 \pm 0.006$ & $3819 \pm 98$ & 27 \\
\hline YY Gem B & $0.599 \pm 0.005$ & $0.619 \pm 0.006$ & $3819 \pm 98$ & 27 \\
\hline
\end{tabular}

References. (1) Hartman et al. 2011, (2) Hełminiak et al. 2012, (3) Fuensalida et al. 1987, (4) Popper 1994, (5) Morales et al. 2009, (6) Ribas 2003, (7) Irwin et al. 2009, (8) Ségransan et al. 2003, (9) Valenti \& Fischer 2005, (10) Demory et al. 2009, (11) López-Morales \& Ribas 2005, (12) Zhou et al. 2014, (13) Torres et al. 2002, (14) Triaud et al. 2013a, (15) Doyle et al. 2011, (16) Ofir et al. 2012, (17) Carter et al. 2011, (18) Irwin et al. 2011, (19) Kraus et al. 2011, (20) LopezMorales et al. 2006, (21) Dimitrov \& Kjurkchieva 2010, (22) Fernandez et al. 2009, (23) Popper 1997, (24) Grundahl et al. 2008, (25) Sandquist et al. 2013, (26) Birkby et al. 2012, (27) Torres \& Ribas 2002.

${ }^{a}$ Data compiled primarily from tables given in Torres et al. (2010), Nefs et al. (2013), and Zhou et al. (2014). We provide the original references for each source in the table.

${ }^{\mathrm{b}}$ This is a single star with an interferometric radius measurement and mass estimated assuming a mass-luminosity relation.

Note that due to the somewhat higher scatter in the stellar parameters at the high mass end and the small number of stars constraining the fit at this end, we find that the stellar mass as a function of density reaches a local maximum of $\sim 0.8 M_{\odot}$ at $\rho \sim 1.5 \mathrm{~g} \mathrm{~cm}^{-3}$. We therefore do not suggest using these models for stars with $M>0.8 M_{\odot}$, or spectral types earlier than $\mathrm{K} 5$.
It is worth comparing our modeling to that done by Johnson et al. (2011, 2012). One significant difference is the treatment of metallicity. Johnson et al. (2011) include a correlation between metallicity and $\Delta M_{K}$ (Johnson \& Apps 2009), while Johnson et al. (2012) include an additional correlation between metallicity and $\Delta J-K$ in their modeling. We choose not to include metallicity explicitly in our model due to the lack of a 
Table 6

K and M Dwarf Stars with Absolute Magnitudes and Measured Masses Used in Fitting the Bolometric Corrections in the Empirical Stellar Model ${ }^{\mathrm{a}}$

\begin{tabular}{|c|c|c|c|c|c|}
\hline Star & $\begin{array}{l}\text { Mass } \\
\left(M_{\odot}\right)\end{array}$ & $\begin{array}{c}M_{V} \\
(\mathrm{mag})\end{array}$ & $\begin{array}{c}M_{J} \\
(\mathrm{mag})\end{array}$ & $\begin{array}{c}M_{H} \\
(\mathrm{mag})\end{array}$ & $\begin{array}{c}M_{K} \\
(\mathrm{mag})\end{array}$ \\
\hline G1 $866 \mathrm{C}$ & $0.0930 \pm 0.0008$ & $17.43 \pm 0.40$ & $\ldots$ & $\ldots$ & $\ldots$ \\
\hline Gl 65 B & $0.100 \pm 0.010$ & $15.87 \pm 0.06$ & $10.06 \pm 0.05$ & $9.45 \pm 0.03$ & $9.16 \pm 0.07$ \\
\hline Gl 65 A & $0.102 \pm 0.010$ & $15.41 \pm 0.05$ & $9.68 \pm 0.05$ & $9.15 \pm 0.03$ & $8.76 \pm 0.07$ \\
\hline G1 234 B & $0.1034 \pm 0.0035$ & $16.16 \pm 0.07$ & $10.31 \pm 0.25$ & $9.56 \pm 0.10$ & $9.26 \pm 0.04$ \\
\hline Gl 623 B & $0.1142 \pm 0.0083$ & $16.02 \pm 0.11$ & $10.47 \pm 0.29$ & $9.35 \pm 0.05$ & $9.33 \pm 0.14$ \\
\hline Gl 866 B & $0.1145 \pm 0.0012$ & $15.64 \pm 0.08$ & $\ldots$ & $9.29 \pm 0.04$ & $8.96 \pm 0.04$ \\
\hline Gl 866 A & $0.1187 \pm 0.0011$ & $15.39 \pm 0.07$ & $\ldots$ & $\ldots$ & $\ldots$ \\
\hline G1 791.2 B & $0.126 \pm 0.003$ & $16.64 \pm 0.10$ & $\ldots$ & $\ldots$ & $\ldots$ \\
\hline Gl 473 B & $0.131 \pm 0.010$ & $15.00 \pm 0.07$ & $9.57 \pm 0.06$ & $9.04 \pm 0.07$ & $8.84 \pm 0.08$ \\
\hline Gl 473 A & $0.143 \pm 0.011$ & $15.01 \pm 0.07$ & $9.44 \pm 0.06$ & $8.84 \pm 0.06$ & $8.40 \pm 0.06$ \\
\hline Gl 831 B & $0.1621 \pm 0.0065$ & $14.62 \pm 0.08$ & $\ldots$ & $8.62 \pm 0.05$ & $8.36 \pm 0.05$ \\
\hline G1 860 B & $0.1762 \pm 0.0066$ & $13.46 \pm 0.09$ & $9.03 \pm 0.08$ & $8.40 \pm 0.05$ & $8.32 \pm 0.07$ \\
\hline G1 747 B & $0.1997 \pm 0.0008$ & $12.52 \pm 0.06$ & $\ldots$ & $\ldots$ & $7.63 \pm 0.04$ \\
\hline G1 $234 \mathrm{~A}$ & $0.2027 \pm 0.0106$ & $13.07 \pm 0.05$ & $8.52 \pm 0.06$ & $7.93 \pm 0.04$ & $7.64 \pm 0.04$ \\
\hline CMDra B & $0.2136 \pm 0.0010$ & $12.94 \pm 0.10$ & $\ldots$ & ... & ... \\
\hline Gl 747 A & $0.2137 \pm 0.0009$ & $12.30 \pm 0.06$ & $\ldots$ & $\ldots$ & $7.53 \pm 0.04$ \\
\hline CMDra A & $0.2307 \pm 0.0010$ & $12.80 \pm 0.10$ & $\ldots$ & $\ldots$ & $\ldots$ \\
\hline Gl $860 \mathrm{~A}$ & $0.2711 \pm 0.0100$ & $11.76 \pm 0.05$ & $7.84 \pm 0.04$ & $7.26 \pm 0.04$ & $6.95 \pm 0.04$ \\
\hline Gl 791.2 A & $0.286 \pm 0.006$ & $13.37 \pm 0.03$ & $\ldots$ & $\ldots$ & $\ldots$ \\
\hline Gl $831 \mathrm{~A}$ & $0.2913 \pm 0.0125$ & $12.52 \pm 0.06$ & $\ldots$ & $7.36 \pm 0.05$ & $7.08 \pm 0.05$ \\
\hline $\mathrm{Gl} 644 \mathrm{Bb}$ & $0.3143 \pm 0.0040$ & $11.71 \pm 0.10$ & $\ldots$ & $\ldots$ & $\ldots$ \\
\hline G1 623 A & $0.3432 \pm 0.0301$ & $10.74 \pm 0.05$ & $7.19 \pm 0.04$ & $6.70 \pm 0.04$ & $6.46 \pm 0.04$ \\
\hline Gl $644 \mathrm{Ba}$ & $0.3466 \pm 0.0047$ & $11.22 \pm 0.10$ & $\ldots$ & $\ldots$ & $\ldots$ \\
\hline Gl 661 B & $0.369 \pm 0.035$ & $11.15 \pm 0.06$ & $7.51 \pm 0.04$ & $7.02 \pm 0.04$ & $6.78 \pm 0.05$ \\
\hline G1 $570 \mathrm{C}$ & $0.3770 \pm 0.0018$ & $11.09 \pm 0.17$ & $7.40 \pm 0.04$ & $6.76 \pm 0.04$ & $6.57 \pm 0.04$ \\
\hline Gl $661 \mathrm{~A}$ & $0.379 \pm 0.035$ & $11.10 \pm 0.06$ & $7.10 \pm 0.05$ & $6.56 \pm 0.04$ & $6.36 \pm 0.05$ \\
\hline GJ $2069 \mathrm{Ab}$ & $0.3987 \pm 0.0007$ & $12.57 \pm 0.19$ & .. & ... & ... \\
\hline Gl 644 A & $0.4155 \pm 0.0057$ & $10.76 \pm 0.06$ & $\ldots$ & $6.61 \pm 0.05$ & $6.35 \pm 0.04$ \\
\hline GJ $2069 \mathrm{Aa}$ & $0.4344 \pm 0.0008$ & $11.78 \pm 0.18$ & $\ldots$ & $\ldots$ & $\ldots$ \\
\hline G1 570 B & $0.5656 \pm 0.0029$ & $9.45 \pm 0.05$ & $6.21 \pm 0.03$ & $5.61 \pm 0.03$ & $5.39 \pm 0.03$ \\
\hline YYGem A & $0.6028 \pm 0.0014$ & $9.03 \pm 0.12$ & $\ldots$ & $\ldots$ & $\ldots$ \\
\hline YYGem B & $0.6069 \pm 0.0014$ & $9.38 \pm 0.14$ & $\ldots$ & $\ldots$ & $\ldots$ \\
\hline HD 195987 B & $0.6650 \pm 0.0079$ & $7.91 \pm 0.19$ & $\ldots$ & $4.835 \pm 0.059$ & $4.702 \pm 0.034$ \\
\hline G1 702 B & $0.713 \pm 0.029$ & $7.52 \pm 0.05$ & $5.63 \pm 0.05$ & $\ldots$ & $4.53 \pm 0.04$ \\
\hline$\chi$ Dra B & $0.750 \pm 0.02$ & $6.11 \pm 0.27$ & $\ldots$ & $\ldots$ & $\ldots$ \\
\hline GJ 765.2 B & $0.763 \pm 0.019$ & $6.64 \pm 0.05$ & $4.94 \pm 0.22$ & $\ldots$ & $4.34 \pm 0.22$ \\
\hline GJ 765.2 A & $0.831 \pm 0.020$ & $5.99 \pm 0.04$ & $4.40 \pm 0.09$ & $\ldots$ & $3.92 \pm 0.09$ \\
\hline HD 195987 A & $0.844 \pm 0.018$ & $5.511 \pm 0.028$ & .. & $3.679 \pm 0.037$ & $3.646 \pm 0.033$ \\
\hline
\end{tabular}

${ }^{a}$ Data taken from Delfosse et al. (2000) and Torres et al. (2010).

large sample of $\mathrm{M}$ dwarfs with well-measured masses, radii, and metallicities. Lacking such a data set, it is not possible to determine the effect of metallicity on all of the relations considered. Instead the metallicity is assumed to contribute to the intrinsic uncertainty in the $\rho \rightarrow R$ and $M \rightarrow M_{\text {bol }}$ relations, measured using the $S_{R}$ and $S_{\text {Mbol }}$ parameters. While, like us, Johnson et al. (2011) use eclipsing binaries in calibrating the mass-radius relation, their sample is the Ribas (2006) catalog which contained only 14 stars with $M<0.8 M_{\odot}$, of which only 10 had masses and radii measured to better than $5 \%$ precision. Johnson et al. (2012), on the other hand, choose to use stars with interferometrically measured radii from Boyajian et al. (2012) due to the possibility that the eclipsing binaries are systematically inflated compared to single stars. While these stars have directly measured radii, their masses must be estimated using an assumed mass-luminosity relation. The few long period, low-mass eclipsing binaries which have been studied have parameters that are consistent with the shorter period binaries (Doyle et al. 2011; Irwin et al. 2011). Moreover Boyajian et al. (2012) find no systematic difference between the mass-radius relation determined from their single stars (for an assumed mass-luminosity relation), and the mass-radius relation determined from short period eclipsing binaries, so we choose to use the eclipsing binaries for which both masses and radii are measured directly.

Having established our empirical stellar model, we use it to determine the properties of HATS- 6 by incorporating it directly into our light curve and RV curve fit. To do this we add the following terms to our likelihood function:

$$
\begin{aligned}
& -\frac{1}{2} \sum_{\lambda}\left(\frac{M_{\lambda}\left(l \rho, d l R, d M_{\mathrm{bol}}\right)+\Delta d+A_{\lambda}-m_{\lambda}}{\sigma_{\lambda}}\right)^{2} \\
& -\frac{1}{2}\left(\left(\frac{d l R}{S_{R}}\right)^{2}+\left(\frac{d M_{\mathrm{bol}}}{S_{M}}\right)^{2}\right)
\end{aligned}
$$

where $M_{\lambda}$ is the predicted absolute magnitude in filter $\lambda$ given $d l R, d M_{\mathrm{bol}}$, and $l \rho$, which is determined from the other free parameters in the model $\left(b^{2}, \zeta / R_{\star}, P, \sqrt{e} \sin \omega, \sqrt{e} \cos \omega\right.$, and 
Table 7

Fitted Parameters of Empirical Model for K and M Dwarf Properties

\begin{tabular}{|c|c|c|}
\hline Parameter & Best Fit Value $^{a}$ & Median and $1 \sigma$ Uncertainty $^{\mathrm{b}}$ \\
\hline$a_{\rho, 0}$ & 0.029 & $0.054_{-0.049}^{+0.048}$ \\
\hline$a_{\rho, 1}$ & -0.2924 & $-0.2989_{-0.0084}^{+0.0083}$ \\
\hline$a_{\rho, 2}$ & 0.0817 & $0.0815_{-0.0020}^{+0.0020}$ \\
\hline$a_{\rho, 3}$ & -0.18187 & $-0.18280_{-0.00074}^{+0.00075}$ \\
\hline$b_{m, 0}$ & 35.3 & $32.6_{-3.3}^{+3.1}$ \\
\hline$b_{m, 1}$ & -7.54 & $-7.09_{-0.44}^{+0.44}$ \\
\hline$b_{m, 2}$ & 1.17 & $1.24_{-0.11}^{+0.10}$ \\
\hline$b_{m, 3}$ & -7.717 & $-7.690_{-0.034}^{+0.034}$ \\
\hline$c_{V, 0}$ & 1070 & $570_{-370}^{+380}$ \\
\hline$c_{V, 1}$ & 15.3 & $16.5_{-2.9}^{+2.2}$ \\
\hline$c_{V, 2}$ & 0.099 & $0.111_{-0.013}^{+0.013}$ \\
\hline$c_{J, 0}$ & 130 & $100_{-190}^{+150}$ \\
\hline$c_{J, 1}$ & -5.0 & $-5.4_{-1.1}^{+1.2}$ \\
\hline$c_{J, 2}$ & -0.1154 & $-0.1105_{-0.0046}^{+0.0043}$ \\
\hline$c_{H, 0}$ & -70 & $-130_{-140}^{+150}$ \\
\hline$c_{H, 1}$ & -3.96 & $-3.46_{-1.13}^{+0.96}$ \\
\hline$c_{H, 2}$ & -0.1619 & $-0.1615_{-0.0041}^{+0.0044}$ \\
\hline$c_{K, 0}$ & 50 & $-80_{-140}^{+140}$ \\
\hline$c_{K, 1}$ & -5.08 & $-4.83_{-0.99}^{+0.92}$ \\
\hline$c_{K, 2}$ & -0.1786 & $-0.1757_{-0.0038}^{+0.0039}$ \\
\hline$S_{R}$ & 0.0069 & $0.0091_{-0.0012}^{+0.0010}$ \\
\hline$S_{\mathrm{Mbol}}$ & 0.138 & $0.169_{-0.025}^{+0.022}$ \\
\hline
\end{tabular}

${ }^{a}$ A self-consistent set of values corresponding to the maximum likelihood model.

${ }^{\mathrm{b}}$ The median and $1 \sigma$ confidence interval for each parameter as determined from an MCMC analysis.

$K) ; \Delta d$ is the distance modulus and $A_{\lambda}$ is the extinction; $m_{\lambda}$ is the observed magnitude; and $\sigma_{\lambda}$ is the magnitude uncertainty. The sum is over the filters $\lambda=V, J, H$, and $K$. The new parameters introduced in the fit are thus $d l R, d M_{\mathrm{bol}}, \Delta d$, and $A_{V}$. We restrict $A_{V}$ to be in the range [0, 0.175] where the upper limit is the total line-of-sight extinction from the Schlafly \& Finkbeiner (2011) reddening maps, and we assume the Cardelli et al. (1989) extinction law to relate $A_{V}$ to the extinction in the other band-passes. Given $l \rho, d l R$, and $d M_{\mathrm{bol}}$ at each link in the resulting Markov Chain, we then calculate all relevant stellar parameters and dependent planetary parameters.

Using the empirical model we find $M_{\star}=0.574_{-0.027}^{+0.020} M_{\odot}$ and $R_{\star}=0.570 \pm 0.011 R_{\odot}$, assuming a circular orbit, and $M_{\star}=0.573 \pm 0.031 M_{\odot}$ and $R_{\star}=0.567 \pm 0.037 R_{\odot}$, allowing for an eccentric orbit. The mass and radius inferred assuming a circular orbit are slightly lower than, though consistent with, the parameters inferred using the Dartmouth isochrones $\quad\left(M_{\star}=0.595 \pm 0.017 M_{\odot}, \quad\right.$ and $\quad R_{\star}=0.5759$ $\left.\pm 0.0092 R_{\odot}\right)$. The precision allowed by both the Dartmouth and empirical models are similar. When we allow the eccentricity to vary with our empirical model the constraint from the observed $V-K$ color of the star pulls the model to a lower eccentricity solution $(e=0.053 \pm 0.060)$, yielding parameters that are consistent with those from the fixedcircular model. The $95 \%$ and $99 \%$ confidence upper-limits on the eccentricity are $e<0.17$ and $e<0.25$, respectively. For the final parameters we suggest adopting those from the empirical stellar model, assuming a circular orbit. The adopted stellar parameters are listed in Table 9 while the planetary parameters are listed in Table 10.

\section{DISCUSSION}

In this paper we have presented the discovery of the HATS-6 transiting planet system by the HATSouth survey. We found that HATS-6b has a mass of $0.319 \pm 0.070 M_{J}$, radius of 0.998 $\pm 0.019 R_{J}$ and orbits a star with a mass of $0.574_{-0.027}^{+0.020} M_{\odot}$. HATS-6 is one of only four stars with $M \lesssim 0.6 M_{\odot}$ known to host a short period gas giant planet (the other three are WASP-80, WASP-43, and Kepler-45; curiously all are transiting planets; see Table 8). This is illustrated in Figure 11 where we plot planet mass against the host star mass. We note that the published masses for these stars come from a disparate set of methods. Applying the empirical relations developed in this paper (Section 3.3.2) to these systems, using $\rho_{\star}, V, J, H$, and $K_{S}$ as observables, we find masses of $0.614 \pm 0.031 M_{\odot}$, and $0.601 \pm 0.031 M_{\odot}$, for WASP-43 and WASP-80, respectively, slightly higher than the published values of $0.580 \pm 0.050 M_{\odot}$ and $0.596 \pm 0.035 M_{\odot}$. This same method yields a mass of $0.534 \pm 0.034 M_{\odot}$ for Kepler- 45 , which is a bit lower than the published value of $0.570 \pm 0.059 M_{\odot}$.

HATS-6b also has some of the deepest transits known, with $\left(R_{p} / R_{\star}\right)^{2}=0.0323 \pm 0.0003$. There are only two known planets with deeper transits than HATS-6b: Kepler-45b $\left(\left(R_{p} / R_{\star}\right)^{2}=0.0362 \pm 0.0054\right.$; Southworth 2012), and WTS$2 \mathrm{~b}$, a hot Jupiter on a very short period orbit around a K2 dwarf star $\left(\left(R_{p} / R_{\star}\right)^{2}=0.0347 \pm 0.0008\right.$; Birkby et al. 2014). One other system with transit depths close to that of HATS-6 is WASP-80b $\left(\left(R_{p} / R_{\star}\right)^{2}=0.0292 \pm 0.0001 ;\right.$ Mancini et al. 2014). Figure 11 illustrates this as well, plotting the transit depth versus planet mass.

With an early $M$ dwarf host star, HATS-6b receives substantially less stellar irradiation than planets with the same orbital period around Sun-like stars. As a result the equilibrium temperature, assuming zero albedo, is a relatively mild $712.8 \pm 5.1 \mathrm{~K}$. The atmospheric chemistry at this temperature is expected to be notably different from other hotter planets, with a greater abundance of $\mathrm{CH}_{4}$ and associated hydro-carbon hazes (Howe \& Burrows 2012; Fortney et al. 2013). This makes HATS-6b a potentially interesting target for studying the atmospheric properties of a warm gas giant. In the bottom two panels of Figure 11 we plot the approximate $K$-band transmission spectrum $\mathrm{S} / \mathrm{N}$ and $4.5 \mu \mathrm{m}$ occultation spectrum $\mathrm{S} / \mathrm{N}$ versus $T_{\text {eq }}$. In both cases we normalize the $\mathrm{S} / \mathrm{N}$ to that of the prototypical hot Jupiter HD 189733b. For the transmission spectrum S/N we use the relation (e.g., Winn 2010):

$$
\mathrm{S} / \mathrm{N} \propto \frac{2 R_{P} H N_{H}}{R_{\star}^{2}} \sqrt{T_{14} F_{K}}
$$

where $H=k_{B} T_{\mathrm{eq}} / \mu g_{P}, \mu$ is the mean molecular weight (we assume $3.347 \times 10^{-27} \mathrm{~kg}$ for a pure $\mathrm{H}_{2}$ atmosphere), $g_{P}$ is the surface gravity of the planet, $T_{14}$ is the transit duration, $F_{K}$ is the $K$-band flux of the star in Janskys, and $N_{H}$ is a constant of order unity (we assume 1) depending on the particular spectroscopic feature under consideration. The term $\sqrt{T_{14} F_{K}}$ is included because the photometric noise is proportional to $1 / \sqrt{F_{K}}$ and the number of in transit measurements that can be made is proportional to $T_{14}$ (meaning the noise goes as 

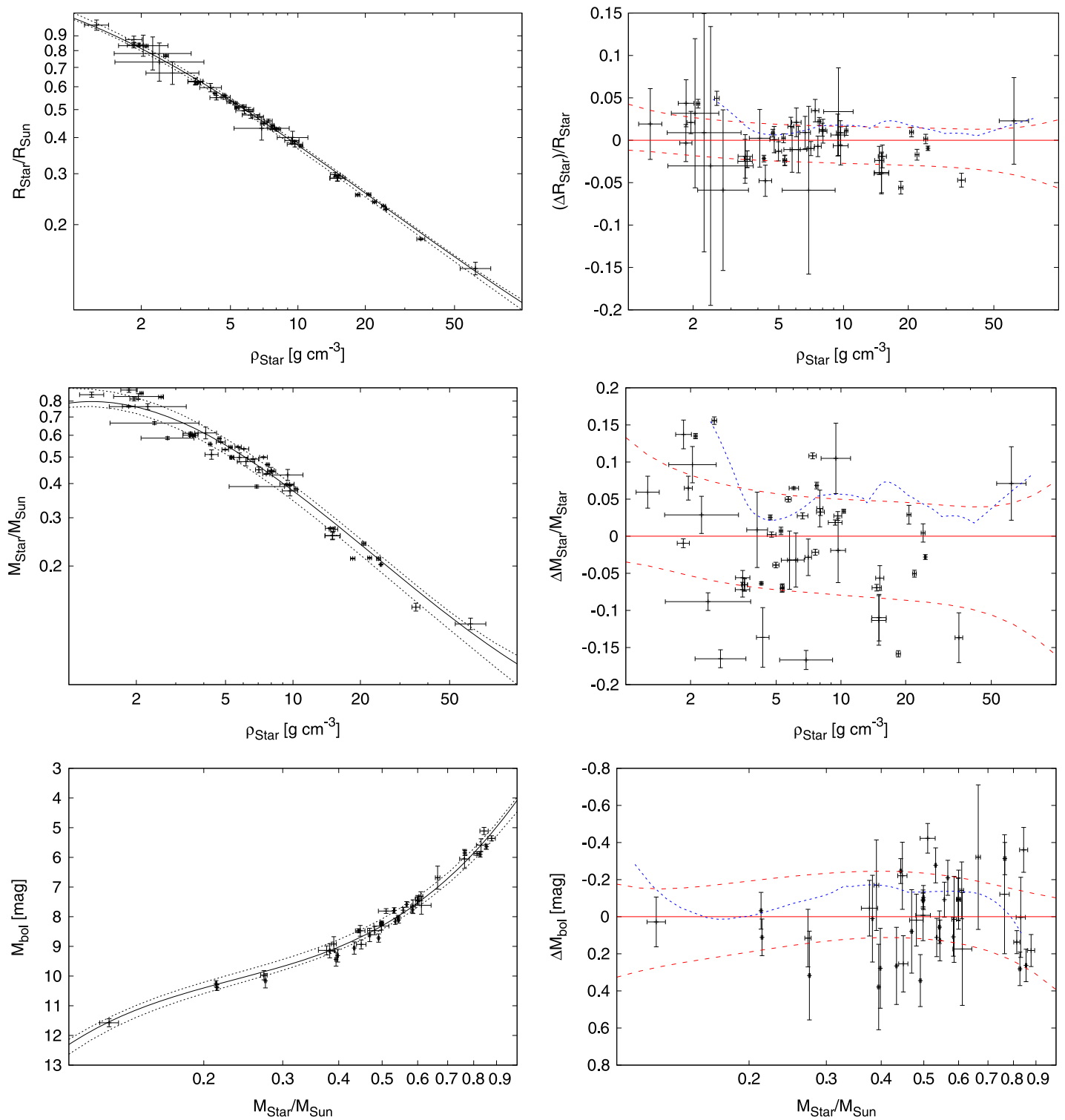

Figure 6. Top: empirical relation between stellar density and radius (Equation (2), with parameters given in Table 7). The solid line shows the best-fit relation with $d R=0$, the dashed lines show the expected $1 \sigma$ spread for this relation allowing $d R$ to be drawn from a Gaussian distribution with standard deviation $S_{R}$. The points show the eclipsing binary components from Table 5 used to fit this relation. Middle: same as top; here we show the empirical relation between stellar density and mass. Bottom: same as top; here we show the empirical relation between stellar mass and bolometric magnitude. On the right-hand panels we show the fractional residuals from the best-fit models, the short-dashed (blue) lines show the expected relations from the Dartmouth stellar models for an age of 4.5 Gyr and solar metallicity, while the longer dashed (red) lines show the expected $1 \sigma$ spread from the empirical model. The solar-metallicity 4.5 Gyr Dartmouth models predict systematically larger radii at fixed density, and brighter bolometric magnitudes at fixed mass, than observed in the eclipsing binary sample. The empirical models provide a good fit to these data by design.

$1 / \sqrt{T_{14}}$.) We neglect the constant of proportionality on the right-hand side of Equation (10) needed to make the expression for $\mathrm{S} / \mathrm{N}$ unitless. For the occultation spectrum $\mathrm{S} / \mathrm{N}$ we use the relation (e.g., Winn 2010):

$$
\mathrm{S} / \mathrm{N} \propto\left(\frac{R_{P}}{R_{\star}}\right)^{2} \frac{\exp \left(h c / k_{B} \lambda T_{\text {eff }}\right)-1}{\exp \left(h c / k_{B} \lambda T_{\mathrm{eq}}\right)-1} \sqrt{T_{14} F_{4.5}}
$$

where $h$ is Planck's constant, $c$ is the speed of light, $k_{B}$ is Boltmann's constant, $T_{\text {eff }}$ is the stellar effective temperature, $\lambda$ is the effective wavelength (we assume $4.5 \mu \mathrm{m}$ ), and $F_{4.5}$ is the $4.5 \mu \mathrm{m}$ flux of the star in Janskys.

We find that HATS-6b has the highest expected S/N transmission spectrum among known gas giant planets with
$T_{\mathrm{eq}}<750 \mathrm{~K}$. Regarding occultations, HATS-6b has the second highest expected $\mathrm{S} / \mathrm{N}$ at $4.5 \mu \mathrm{m}$ among gas giant planets with $T_{\text {eq }}<750 \mathrm{~K}$ after HD 80606b (Laughlin et al. 2009; the expected S/N for HATS-6b is $\sim 50 \%$ that of HD 80606b).

Perhaps the most relevant planet for comparison is WASP$80 \mathrm{~b}$, a hot Jupiter with $T_{\mathrm{eq}}=825 \pm 20 \mathrm{~K}$ (Mancini et al. 2014), with a similar transit depth to HATS-6b, but orbiting a somewhat brighter star. High-precision ground-based photometric transit observations of this system over several optical and near-infrared band-passes have been presented by Mancini et al. (2014) and Fukui et al. (2014), which have provided suggestive evidence for the existence of a haze in the atmosphere. The expected transmission $\mathrm{S} / \mathrm{N}$ for HATS- $6 \mathrm{~b}$ is approximately 0.4 times that of WASP-80b, so measuring the 

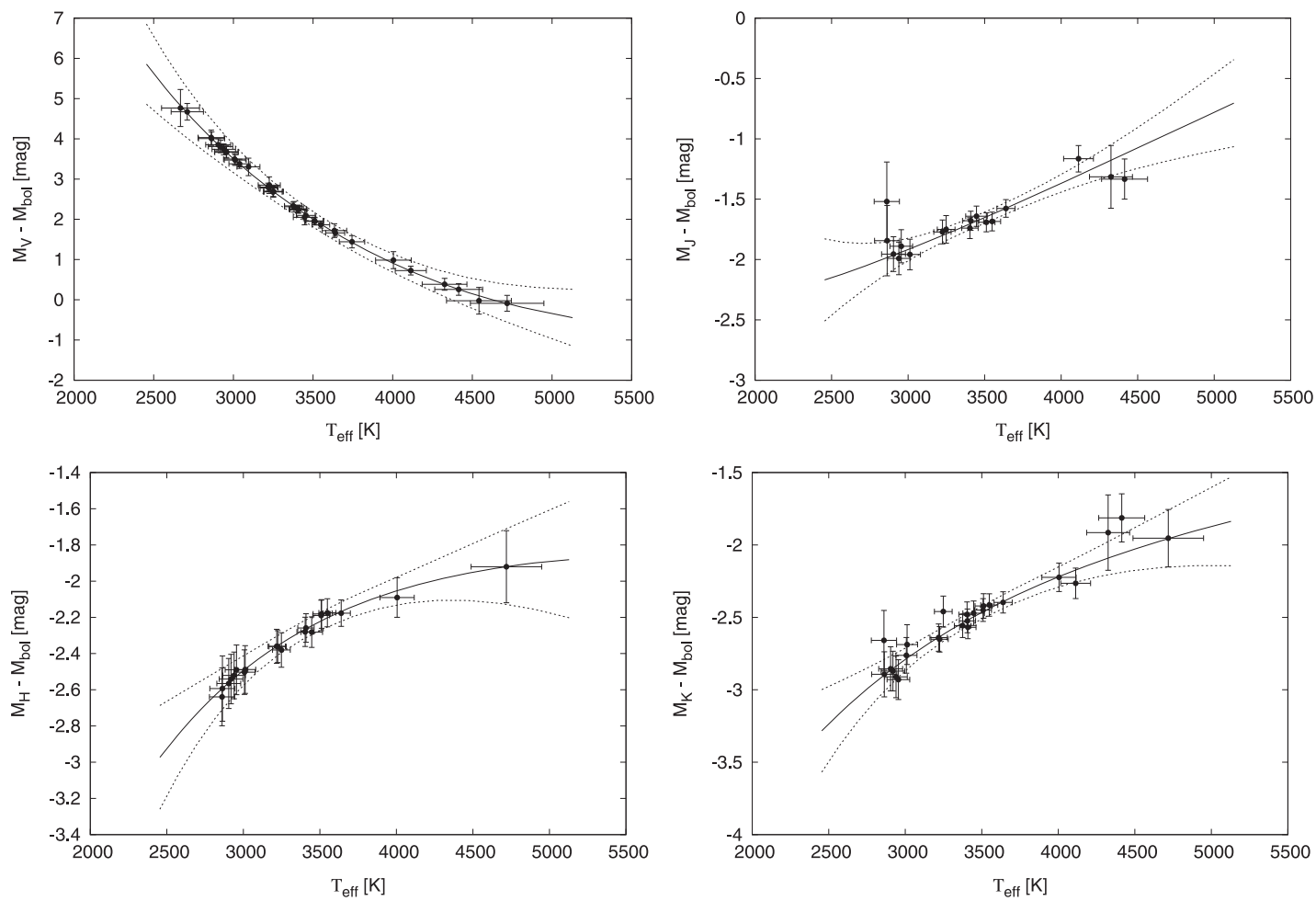

Figure 7. Top: empirical relation between effective temperature and the bolometric correction in $V$-band (upper left), $J$-band (upper right), $H$-band (lower left), and $K$ band (lower right). In each case the solid line is the median relation, while the dashed lines show the $1 \sigma$ confidence region about the median relation. The points in this plot correspond to stars in Table 6 with $T_{\text {eff }}$ and $M_{\mathrm{bol}}$ for each star determined as part of the empirical model fitting procedure. Because $T_{\text {eff }}$ and $M_{\mathrm{bol}}$ are not directly observed parameters for these stars, but are rather determined through the modeling, and largely constrained by the stellar mass, which is observed, the data appear to follow the model very closely. This should not be taken as validation of the model (see instead Figure 8), rather the purpose of showing these plots is to demonstrate the form of the bolometric correction, its uncertainty, as well as the range of temperatures over which the model is constrained.
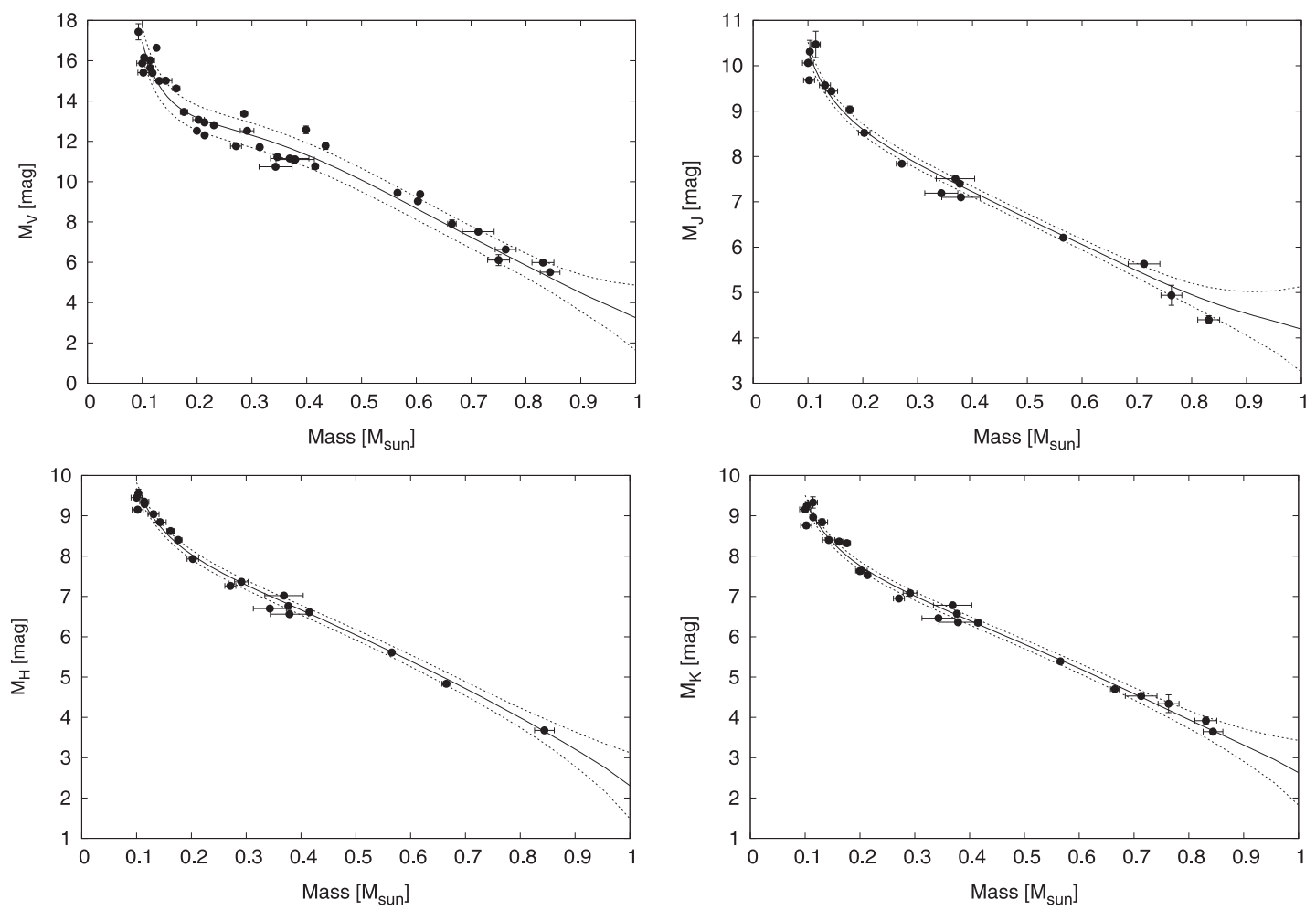

Figure 8. Top: same as Figure 7. Here we show the empirical relation between stellar mass and the absolute magnitudes in the $V, J, H$, and $K$ bands. The residuals from these fits are shown in Figure 9. 

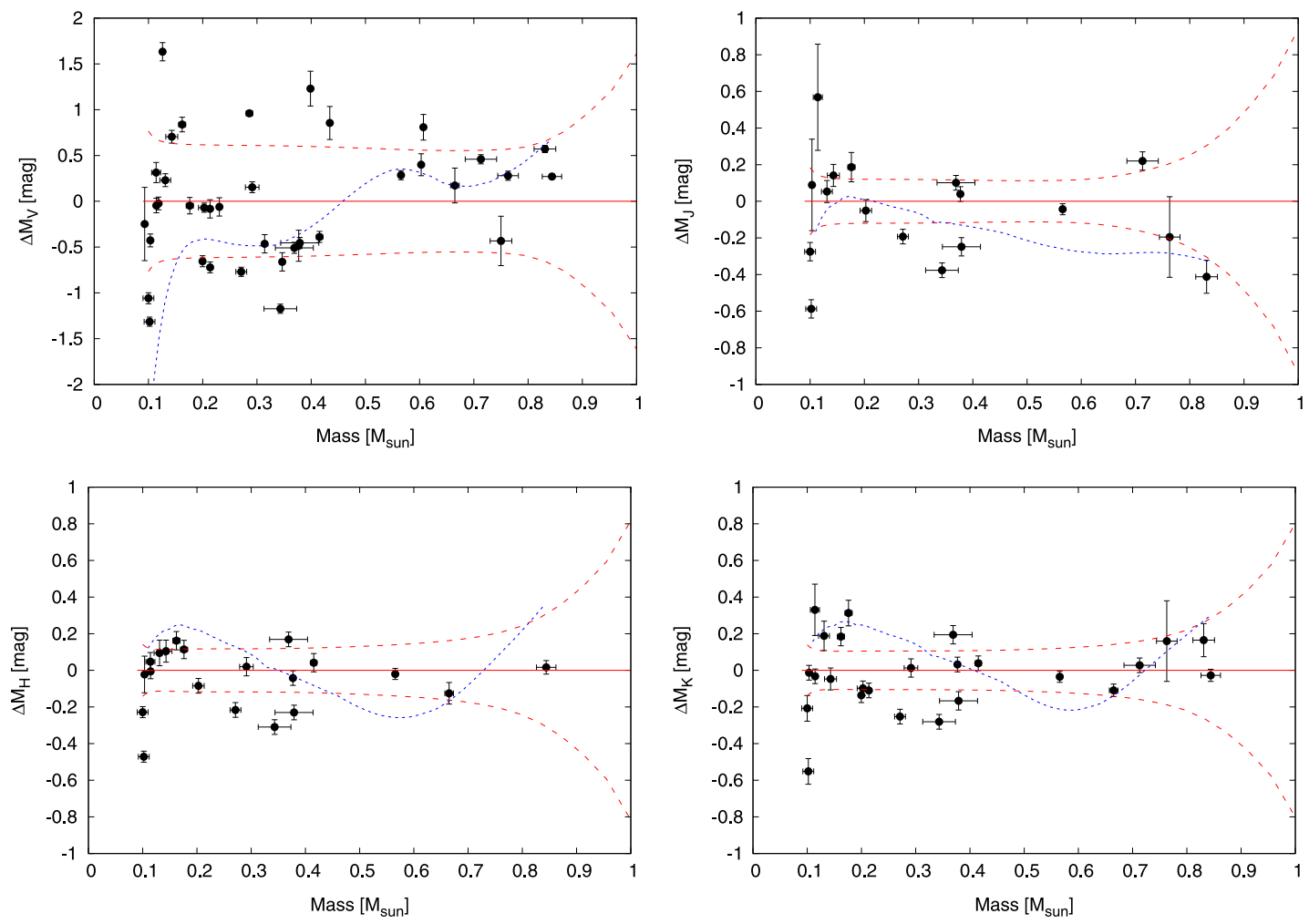

Figure 9. Same as Figure 8. Here we show residuals from the median empirical relation. The short-dash (blue) lines show the relation from the 4.5 Gyr, solar metallicity Dartmouth models, while the long-dash (red) lines show the expected $1 \sigma$ spread of the empirical model.
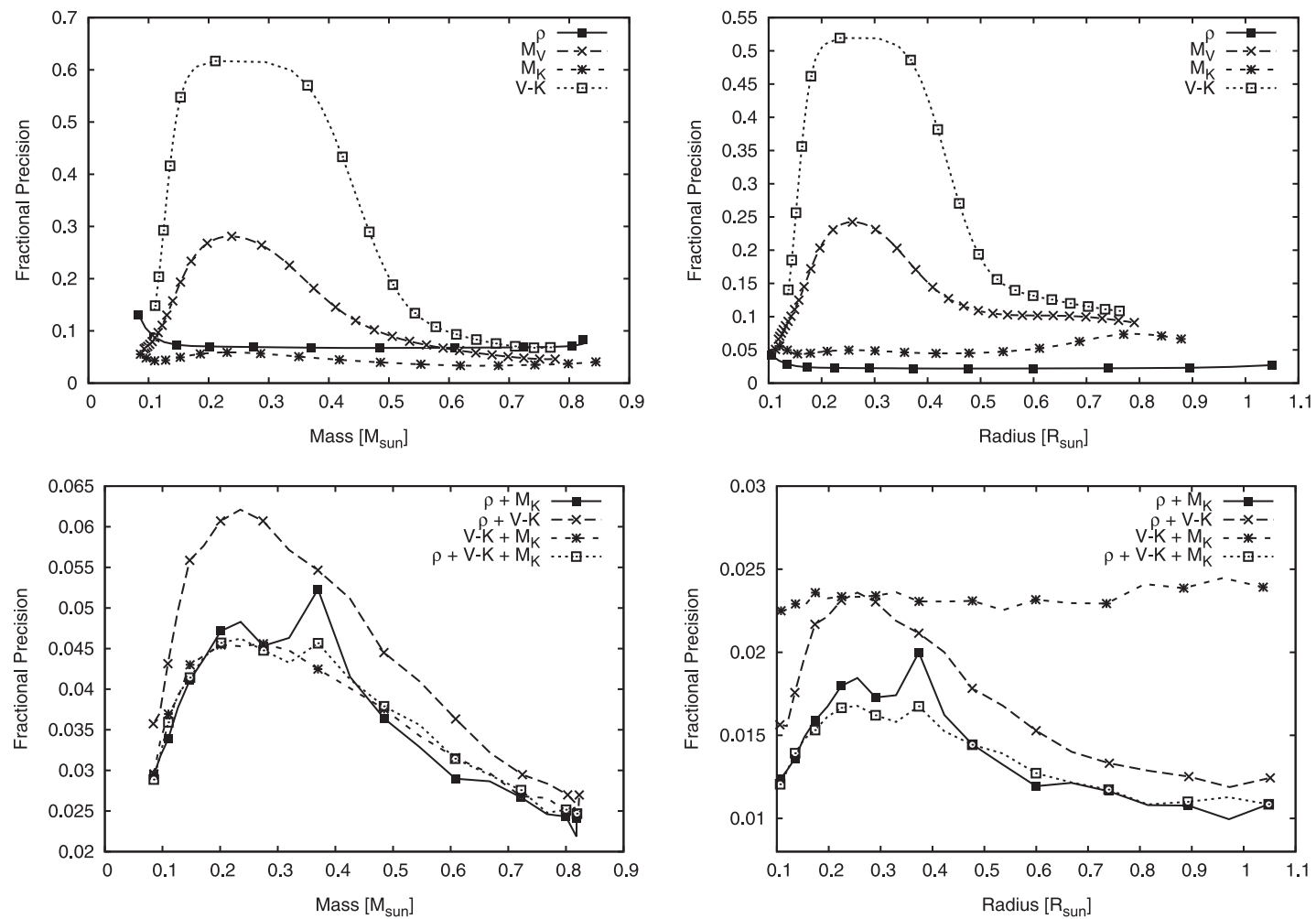

Figure 10. Top: comparison of the precision with which stellar mass (left) and stellar radius (right) may be measured using either $\rho_{\star}, M_{V}, M_{K}$, or $V-K$ as an observed parameter. For each observable we step through a range of values determining the median stellar mass (radius) and its standard deviation using the empirical relations given in Equation (2) through (4). We find that $M_{K}$ is the highest precision stellar mass indicator, allowing a precision that varies between $\sim 3 \%$ and $6 \%$, whereas $\rho_{\star}$ is the highest precision stellar radius indicator, allowing a precision of $\sim 2 \%$ over the range $0.2 R_{\odot}<R<0.9 R_{\odot}$. Bottom: comparison of the precision with which stellar mass (left) and stellar radius (right) may be measured using the combinations of parameters indicated in the figure. Combining the density with an absolute magnitude indicator allows for a mass precision between $2.5 \%$ and $4.5 \%$, and a radius precision between $\sim 1 \%$ and $\sim 2 \%$. 
Table 8

Transiting-planet Hosting Stars with Properties Similar to HATS-6

\begin{tabular}{|c|c|c|c|c|c|c|c|}
\hline Star & $\begin{array}{l}\text { Mass } \\
\left(M_{\odot}\right)\end{array}$ & $\begin{array}{l}\text { Radius } \\
\left(R_{\odot}\right)\end{array}$ & $\begin{array}{r}V-K \\
(\mathrm{mag})\end{array}$ & $\begin{array}{c}\rho_{\star} \\
\left(\mathrm{g} \mathrm{cm}^{-3}\right)\end{array}$ & $\begin{array}{l}\text { Planet Mass } \\
\qquad\left(M_{J}\right)\end{array}$ & $\begin{array}{c}\text { Planet Period } \\
\text { (Day) }\end{array}$ & Reference \\
\hline GJ 3470 & $0.541 \pm 0.067$ & $0.503 \pm 0.063$ & $4.281 \pm 0.030$ & $6.0 \pm 2.4$ & $0.0441 \pm 0.0053$ & 3.34 & 1 \\
\hline KIC 10905746 & $0.578 \pm 0.032$ & $0.548 \pm 0.026$ & $3.94 \pm 0.24$ & $4.97 \pm 0.54$ & $\ldots$ & 9.88 & 3 \\
\hline WASP-43 & $0.580 \pm 0.050$ & $0.598_{-0.042}^{+0.034}$ & $3.212 \pm 0.027$ & $3.81_{-0.51}^{+0.86}$ & $1.78 \pm 0.10$ & 0.81 & 4 \\
\hline Kepler-45 & $0.570 \pm 0.059$ & $0.539 \pm 0.039$ & $3.990 \pm 0.058$ & $5.12 \pm 0.73$ & $0.500 \pm 0.061$ & 2.46 & 5,9 \\
\hline HAT-P-54 & $0.645 \pm 0.020$ & $0.617 \pm 0.013$ & $3.179 \pm 0.063$ & $3.88 \pm 0.27$ & $0.760 \pm 0.032$ & 3.80 & 6 \\
\hline
\end{tabular}

References. (1) Bonfils et al. (2012), (2) Triaud et al. (2013b), (3) Fischer et al. (2012), (4) Hellier et al. (2011), (5) Johnson et al. (2012), (6) Bakos et al. (2014), (7) Steffen et al. (2012), (8) Mancini et al. (2014), (9) Southworth (2012).

${ }^{\mathrm{a}}$ The period listed is for Kepler-26b, the innermost of the four planets transiting this star.
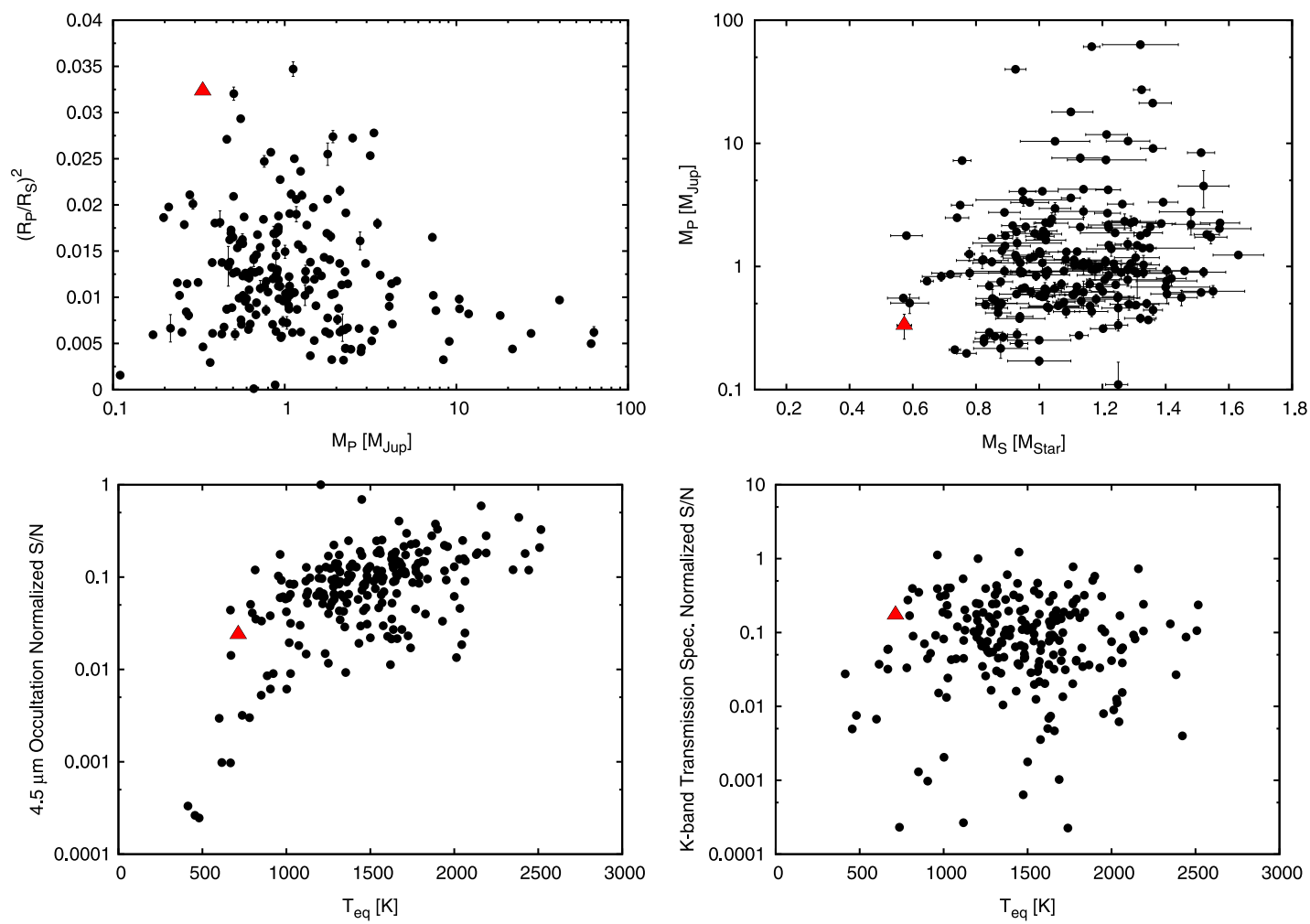

Figure 11. Comparison of the HATS-6 system to other known transiting exoplanet systems with $M_{P}>0.1 M_{J}$. In each case HATS-6 is indicated by the filled red triangle, while other objects are indicated by filled black circles. Top left: planet mass vs. transit depth as measured by $\left(R_{p} / R_{\star}\right)^{2}$. Top right: host star mass vs. planet mass. Bottom left: estimated S/N of a single occultation event at $4.5 \mu \mathrm{m}$ normalized to that of HD $189733 \mathrm{~b}$. Bottom right: estimated S/N of a transmission spectrum signal from one transit, assuming a pure $\mathrm{H}_{2}$ atmosphere, and observed at $K$-band, normalized to that of HD 189733b. HATS-6 has some of the deepest transits of known transiting planet systems, it is also one of the lowest mass stars known to host a transiting gas giant planet. In terms of atmospheric characterization it is one of the highest $\mathrm{S} / \mathrm{N}$ targets with $T_{\mathrm{eq}}<750 \mathrm{~K}$ for both occultation and transmission spectroscopy.

transmission spectrum for HATS-6b will be challenging, but not out of reach for ground-based facilities. HATS-6b is also a promising target for the NASA James Webb Space Telescope mission, currently scheduled for launch in 2018.

We checked whether the simultaneous optical multi-filter GROND observations that we have already obtained for HATS- 6 can be used to probe the transmission spectrum. We fit a Mandel \& Agol (2002) transit model to each of the GROND $g, r, i$, and $z$ light curves, fixing all transit parameters to the best-fit values for the system, except for $R_{p} / R_{\star}$, which we allow to vary independently for each band-pass. We find that values for $R_{p} / R_{\star}$ values are consistent with a flat transmission spectrum, to within the uncertainties. Additional observations would be needed to detect spectroscopic features in the atmosphere of HATS- 6 b.

In order to characterize the host star HATS-6 we have also presented a new set of empirical stellar models applicable to main-sequence stars with $M<0.80 M_{\odot}$. We use eclipsing binaries as well as resolved binaries with masses and measured absolute magnitudes to determine empirical relations between $\rho_{\star}$ and $R$ (and thus $\rho_{\star}$ and $M$ ), between $M$ and $M_{\mathrm{bol}}$, and between $T_{\text {eff }}$ and the $V, J, H$, and $K$-band bolometric corrections. We perform a global fit to the available data, determining the intrinsic scatter in the relations in a selfconsistent manner. We find that from the density alone it is possible to measure the mass of a $\sim 0.6 M_{\odot}$ star to $\sim 7 \%$ 
Table 9

Stellar Parameters for HATS-6

\begin{tabular}{|c|c|c|c|}
\hline Parameter & $\begin{array}{c}\text { Value Isochrones }{ }^{\mathrm{a}} \\
\left({ }^{\circ}\right)\end{array}$ & $\begin{array}{c}\text { Value Empirical }{ }^{\mathrm{b}} \\
\left({ }^{\circ}\right) \\
\end{array}$ & $\begin{array}{c}\text { Value Empirical }^{\mathrm{b}} \\
\text { Eccen. }\end{array}$ \\
\hline \multicolumn{4}{|l|}{ Identifying Information } \\
\hline R.A. (h:m:s; 2MASS) & $05^{\mathrm{h}} 52^{\mathrm{m}} 35.22 \mathrm{~s}$ & $\ldots$ & $\ldots$ \\
\hline Decl. (d:m:s; 2MASS) & $-19^{\circ} 01^{\prime} 54.0^{\prime \prime}$ & $\ldots$ & $\ldots$ \\
\hline 2MASS ID & 2MASS 05523523-1901539 & $\ldots$ & $\ldots$ \\
\hline \multicolumn{4}{|l|}{ Identifying Information } \\
\hline$T_{\text {eff } \star}(\mathrm{K})$ & $3770 \pm 100$ & $\cdots$ & $\cdots$ \\
\hline$[\mathrm{Fe} / \mathrm{H}]$ & $0.200 \pm 0.091$ & $\ldots$ & $\ldots$ \\
\hline \multicolumn{4}{|l|}{ Identifying Information } \\
\hline$B(\mathrm{mag} ;$ APASS $)$ & $16.664 \pm 0.043$ & $\ldots$ & $\ldots$ \\
\hline$V$ (mag; APASS) & $15.160 \pm 0.024$ & $\ldots$ & $\ldots$ \\
\hline$J$ (mag; 2MASS) & $12.046 \pm 0.024$ & $\ldots$ & $\ldots$ \\
\hline$H(\mathrm{mag} ; 2 \mathrm{MASS})$ & $11.397 \pm 0.023$ & $\ldots$ & $\ldots$ \\
\hline$K_{s}(\mathrm{mag} ; 2 \mathrm{MASS})$ & $11.224 \pm 0.023$ & $\ldots$ & $\ldots$ \\
\hline \multicolumn{4}{|l|}{ Identifying Information } \\
\hline$M_{\star}(\odot)$ & $0.595 \pm 0.017$ & $0.574_{-0.027}^{+0.020}$ & $0.573 \pm 0.031$ \\
\hline$R_{\star}\left(R_{\odot}\right)$ & $0.5759 \pm 0.0092$ & $0.570 \pm 0.011$ & $0.567 \pm 0.037$ \\
\hline$T_{\text {eff } \star}(\mathrm{K})^{\mathrm{d}}$ & $3872 \pm 53$ & $3724 \pm 18$ & $3722 \pm 19$ \\
\hline$\rho_{\star}(\mathrm{cgs})$ & $4.40 \pm 0.13$ & $4.36 \pm 0.15$ & $4.42_{-0.47}^{+0.75}$ \\
\hline $\log g_{\star}(\mathrm{cgs})$ & $4.6920 \pm 0.0077$ & $4.683 \pm 0.010$ & $4.689 \pm 0.038$ \\
\hline$L_{\star}\left(L_{\odot}\right)$ & $0.0670 \pm 0.0054$ & $0.0558 \pm 0.0024$ & $0.0555 \pm 0.0073$ \\
\hline$M_{V}(\mathrm{mag})$ & $9.43 \pm 0.19$ & $9.209 \pm 0.075$ & $9.22 \pm 0.15$ \\
\hline$M_{K}(\mathrm{mag}, \mathrm{ESO})$ & $5.079 \pm 0.065$ & $5.387 \pm 0.043$ & $5.40 \pm 0.14$ \\
\hline Age (Gyr) & $8.1 \pm 4.3$ & $\ldots$ & $\ldots$ \\
\hline Distance (pc) & $172.6 \pm 5.5$ & $148.4 \pm 3.3$ & $147.8_{-9.4}^{+7.0}$ \\
\hline
\end{tabular}

\footnotetext{
${ }^{a}$ Parameters based on combining the bulk stellar density determined from our fit to the light curves and RV data for HATS-6, the effective temperature and metallicity from the Magellan/PFS spectrum, together with the Dartmouth (Dotter et al. 2008) stellar evolution models. We perform the fit two ways: allowing the eccentricity to vary (Eccen.), and keeping it fixed to zero (Circ.). The estimated value for $\rho_{\star}$ differs significantly between these two fits. For the eccentric orbit fit the stellar density cannot be reproduced by the Dartmouth models given the measured effective temperature and metallicity. We therefore only list the parameters based on the Dartmouth models for the circular orbit fit.

${ }^{\mathrm{b}}$ Parameters based on our empirical relations for $\mathrm{K}$ and $\mathrm{M}$ dwarf stellar properties, which are included directly in our global modeling of the light curves and RV data for HATS-6. Again we perform the fit two ways: allowing the eccentricity to vary, and keeping it fixed to zero. In this case including the constraint from the observed $V-K$ color of the star directly in the RV and light-curve modeling pulls the free-eccentricity model to a lower eccentricity solution, yielding stellar parameters that are consistent with those from the fixed-circular model.

${ }^{c}$ These parameters are determined from the $\mathrm{I}_{2}$-free Magellan/PFS spectrum of HATS-6 using the method of Neves et al. (2014).

$\mathrm{d}$ The effective temperature listed here is derived from the Dartmouth stellar models, or from our empirical stellar relations, and is not measured directly from the spectrum.
}

precision, and the radius to $\sim 2 \%$ precision. These precisions may be improved by up to a factor of two, to $\sim 3 \%$ and $\sim 1 \%$, respectively, by incorporating additional photometric data. The relations presented here are similar to those presented by Johnson et al. (2011) and Johnson et al. (2012) except that we do not attempt to incorporate metallicity directly into the model (it contributes instead to the inherent scatter in the relations), and we use a much larger sample of eclipsing binaries than previously considered. Future improvements to our model may be made by incorporating metallicity information directly into the model, but this will require a large sample of stars with measured masses, radii, absolute magnitudes, and metallicities.

Finally, we note that the discovery of HATS- $6 \mathrm{~b}$ is a demonstration of the enhanced sensitivity of HATSouth to planets around low-mass $\mathrm{K}$ and $\mathrm{M}$ dwarf stars relative to other wide-field ground-based surveys, such as HATNet or WASP. The reason for this enhanced sensitivity is the larger aperture optics used by HATSouth, providing higher photometric precision for fainter stars. HATSouth yields better than $2 \%$ precision, per $240 \mathrm{~s}$ integration, for stars down to $r \sim 14.5$. For comparison, if using the same integration time as HATSouth, HATNet would yield $2 \%$ precision for stars with $r \lesssim 13$. Based on the TRILEGAL Galactic models (Girardi et al. 2005) we estimate that there are $\sim 230,000$ stars with $M<0.6 M_{\odot}$, $r<14.5$ in the southern sky and at least $10^{\circ}$ from the Galactic disk that could be observed by HATSouth. This is nearly a factor of 10 more than the number of stars with $M<0.6 M_{\odot}$. and $r<13.0$ in the northern sky that could be observed by HATNet to the same photometric precision. While in principle the discovery of HATS-6b may be used to infer the occurrence rate of hot Jupiters around early M dwarfs (e.g., Gaidos \& Mann 2014), in practice this requires a careful calibration of our transit detection efficiency over the sample of M dwarfs that we have observed. This is beyond the scope of the present paper, but will be the subject of future work.

Development of the HATSouth project was funded by NSF MRI grant NSF/AST-0723074, operations have been supported by NASA grants NNX09AB29G and NNX12AH91H, and follow-up observations receive partial support from grant NSF/ 
Table 10

Orbital and Planetary Parameters

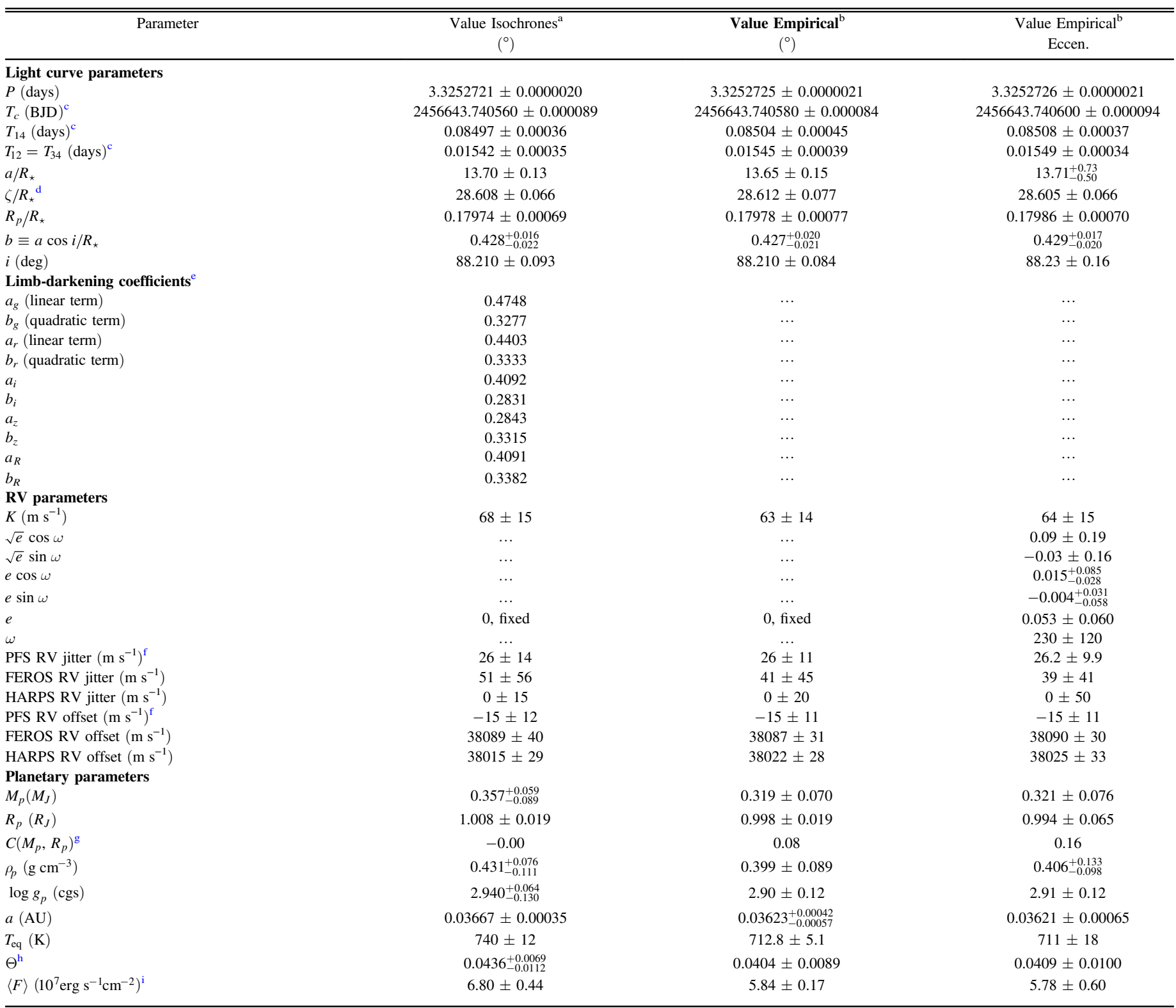

${ }^{\text {a }}$ Parameters based on combining the bulk stellar density determined from our fit to the light curves and RV data for HATS-6, the effective temperature and metallicity from the Magellan/PFS spectrum, together with the Dartmouth (Dotter et al. 2008) stellar evolution models. We perform the fit two ways: allowing the eccentricity to vary (Eccen.), and keeping it fixed to zero (Circ.). The estimated value for $\rho_{\star}$ differs significantly between these two fits. For the eccentric orbit fit the stellar density cannot be reproduced by the Dartmouth models given the measured effective temperature and metallicity. We therefore only list the parameters based on the Dartmouth models for the circular orbit fit.

${ }^{\mathrm{b}}$ Parameters based on our empirical relations for $\mathrm{K}$ and $\mathrm{M}$ dwarf stellar properties, which are included directly in our global modeling of the light curves and RV data for HATS-6. Again we perform the fit two ways: allowing the eccentricity to vary, and keeping it fixed to zero. In this case including the constraint from the observed $V-K$ color of the star directly in the RV and light-curve modeling pulls the free-eccentricity model to a lower eccentricity solution, yielding parameters that are consistent with those from the fixed-circular model. We adopt the empirical models, with fixed circular orbit, for our final system parameters.

${ }^{\mathrm{c}} T_{c}$ : reference epoch of mid transit that minimizes the correlation with the orbital period. BJD is calculated from UTC. $T_{14}$ : total transit duration, time between first to last contact; $T_{12}=T_{34}$ : ingress/egress time, time between first and second, or third and fourth contact.

${ }^{\mathrm{d}}$ Reciprocal of the half duration of the transit used as a jump parameter in our MCMC analysis in place of $a / R_{\star}$. It is related to $a / R_{\star}$ by the expression $\zeta / R_{\star}=a / R_{\star}(2 \pi(1+e \sin \omega)) /\left(P \sqrt{1-b^{2}} \sqrt{1-e^{2}}\right)($ Bakos et al. 2010).

${ }^{\mathrm{e}}$ Values for a quadratic law given separately for the Sloan $g, r, i$, and $z$ filters, as well as for the $R$ filter. These values were adopted from the tabulations by Claret (2004) assuming $T_{\text {eff }}=3700 \mathrm{~K}$ and $[\mathrm{Fe} / \mathrm{H}]=0$. We used the same limb darkening coefficients for all four of the models shown here.

${ }^{\mathrm{f}}$ The jitter was added in quadrature to the RV uncertainties and varied in the fit following (Hartman et al. 2012). We assumed an independent jitter for each instrument.

${ }^{\mathrm{g}}$ Correlation coefficient between the planetary mass $M_{p}$ and radius $R_{p}$.

${ }^{\mathrm{h}}$ The Safronov number is given by $\Theta=\frac{1}{2}\left(V_{\mathrm{esc}} / V_{\mathrm{orb}}\right)^{2}=\left(a / R_{p}\right)\left(M_{p} / M_{\star}\right)$ (see Hansen \& Barman 2007).

${ }^{\mathrm{i}}$ Incoming flux per unit surface area, averaged over the orbit. 
AST-1108686. J.H. acknowledges support from NASA grant NNX14AE87G. K.P. acknowledges support from NASA grant NNX13AQ62G. A.J. acknowledges support from FONDECYT project 1130857, BASAL CATA PFB-06, and project IC120009 "Millennium Institute of Astrophysics (MAS)" of the Millenium Science Initiative, Chilean Ministry of Economy. R.B. and N.E. are supported by CONICYT-PCHA/ Doctorado Nacional. R.B. and N.E. acknowledge additional support from project IC120009 "Millenium Institute of Astrophysics (MAS)" of the Millennium Science Initiative, Chilean Ministry of Economy. V.S. acknowledges support form BASAL CATA PFB-06. M.R. acknowledges support from FONDECYT postdoctoral fellowship 3120097. This work is based on observations made with ESO Telescopes at the La Silla Observatory. This paper also uses observations obtained with facilities of the Las Cumbres Observatory Global Telescope. Work at the Australian National University is supported by ARC Laureate Fellowship Grant FL0992131. We acknowledge the use of the AAVSO Photometric All-sky Survey (APASS), funded by the Robert Martin Ayers Sciences Fund, and the SIMBAD database, operated at CDS, Strasbourg, France. Operations at the MPG $2.2 \mathrm{~m}$ Telescope are jointly performed by the Max Planck Gesellschaft and the European Southern Observatory. The imaging system GROND has been built by the high-energy group of MPE in collaboration with the LSW Tautenburg and ESO. We thank Régis Lachaume for his technical assistance during the observations at the MPG $2.2 \mathrm{~m}$ Telescope. We thank Helmut Steinle and Jochen Greiner for supporting the GROND observations presented in this manuscript. We are grateful to P. Sackett for her help in the early phase of the HATSouth project.

\section{REFERENCES}

Adams, F. C., \& Laughlin, G. 2006, ApJ, 649, 1004

Bakos, G. Á., Csubry, Z., Penev, K., et al. 2013, PASP, 125, 154

Bakos, G. Á., Hartman, J. D., Bhatti, W., et al. 2014, arXiv:1404.4417

Bakos, G. Á., Hartman, J. D., Torres, G., et al. 2012, AJ, 144, 19

Bakos, G. Á., Torres, G., Pál, A., et al. 2010, ApJ, 710, 1724

Bayliss, D., Zhou, G., Penev, K., et al. 2013, AJ, 146, 113

Bedford, D. K., Fuensalida, J. J., \& Arevalo, M. J. 1987, A\&A, 182, 264

Birkby, J., Nefs, B., Hodgkin, S., et al. 2012, MNRAS, 426, 1507

Birkby, J. L., Cappetta, M., Cruz, P., et al. 2014, MNRAS, 440, 1470

Bonfils, X., Gillon, M., Udry, S., et al. 2012, A\&A, 546, A27

Boyajian, T. S., von Braun, K., van Belle, G., et al. 2012, ApJ, 757, 112

Brown, T. M., Baliber, N., Bianco, F. B., et al. 2013, PASP, 125, 1031

Butler, R. P., Marcy, G. W., Williams, E., et al. 1996, PASP, 108, 500

Cardelli, J. A., Clayton, G. C., \& Mathis, J. S. 1989, ApJ, 345, 245

Carter, J. A., Fabrycky, D. C., Ragozzine, D., et al. 2011, Sci, 331, 562

Claret, A. 2004, A\&A, 428, 1001

Coelho, P., Barbuy, B., Meléndez, J., Schiavon, R. P., \& Castilho, B. V. 2005 , A\&A, 443, 735

Crane, J. D., Shectman, S. A., Butler, R. P., et al. 2010, SPIE Conf., 7735, 773553

Deeg, H. J., \& Doyle, L. R. 2001, in Third Workshop on Photometry, ed. W. J. Borucki, \& L. E. Lasher (NASA Tech. Report 20010050209; Moffet Field, CA: NASA Ames Research Center), 85

Delfosse, X., Forveille, T., Ségransan, D., et al. 2000, A\&A, 364, 217

Demory, B.-O., Ségransan, D., Forveille, T., et al. 2009, A\&A, 505, 205

Dimitrov, D. P., \& Kjurkchieva, D. P. 2010, MNRAS, 406, 2559

Dopita, M., Hart, J., McGregor, P., et al. 2007, Ap\&SS, 310, 255

Dotter, A., Chaboyer, B., \& Jevremović, D. 2008, ApJS, 178, 89

Doyle, L. R., Carter, J. A., Fabrycky, D. C., et al. 2011, Sci, 333, 1602

Eastman, J., Gaudi, B. S., \& Agol, E. 2013, PASP, 125, 83

Edelson, R. A., \& Krolik, J. H. 1988, ApJ, 333, 646

Enoch, B., Collier Cameron, A., Parley, N. R., \& Hebb, L. 2010, A\&A, 516, A 33
Feiden, G. A., Chaboyer, B., \& Dotter, A. 2011, ApJL, 740, L25

Fernandez, J. M., Latham, D. W., Torres, G., et al. 2009, ApJ, 701, 764

Fischer, D. A., Schwamb, M. E., Schawinski, K., et al. 2012, MNRAS, 419, 2900

Fortney, J. J., Mordasini, C., Nettelmann, N., et al. 2013, ApJ, 775, 80

Fukui, A., Kawashima, Y., Ikoma, M., et al. 2014, ApJ, 790, 108

Gaidos, E., \& Mann, A. W. 2014, ApJ, 791, 54

Girardi, L., Groenewegen, M. A. T., Hatziminaoglou, E., \& da Costa, L. 2005, A\&A, 436, 895

Greiner, J., Bornemann, W., Clemens, C., et al. 2008, PASP, 120, 405

Grundahl, F., Clausen, J. V., Hardis, S., \& Frandsen, S. 2008, A\&A, 492, 171

Hansen, B. M. S., \& Barman, T. 2007, ApJ, 671, 861

Hartman, J. D., Bakos, G. Á, Béky, B., et al. 2012, AJ, 144, 139

Hartman, J. D., Bakos, G. Á, Noyes, R. W., et al. 2011, AJ, 141, 166

Haywood, M. 2001, MNRAS, 325, 1365

Hellier, C., Anderson, D. R., Collier Cameron, A., et al. 2011, A\&A, 535, L7

Hełminiak, K. G., Konacki, M., RóŻyczka, M., et al. 2012, MNRAS, 425,1245

Howard, A. W., Marcy, G. W., Bryson, S. T., et al. 2012, ApJS, 201, 15

Howe, A. R., \& Burrows, A. S. 2012, ApJ, 756, 176

Irwin, J., Charbonneau, D., Berta, Z. K., et al. 2009, ApJ, 701, 1436

Irwin, J. M., Quinn, S. N., Berta, Z. K., et al. 2011, ApJ, 742, 123

Johnson, J. A., Aller, K. M., Howard, A. W., \& Crepp, J. R. 2010, PASP, 122,905

Johnson, J. A., \& Apps, K. 2009, ApJ, 699, 933

Johnson, J. A., Apps, K., Gazak, J. Z., et al. 2011, ApJ, 730, 79

Johnson, J. A., Gazak, J. Z., Apps, K., et al. 2012, AJ, 143, 111

Jordán, A., Brahm, R., Bakos, G. Á, et al. 2014, AJ, 148, 29

Kaufer, A., \& Pasquini, L. 1998, SPIE, 3355, 844

Kovács, G., Bakos, G., \& Noyes, R. W. 2005, MNRAS, 356, 557

Kovács, G., Zucker, S., \& Mazeh, T. 2002, A\&A, 391, 369

Kraus, A. L., Tucker, R. A., Thompson, M. I., Craine, E. R., \& Hillenbrand, L. A. 2011, ApJ, 728, 48

Kurtz, D. W. 1985, MNRAS, 213, 773

Laughlin, G., Deming, D., Langton, J., et al. 2009, Natur, 457, 562

Lopez-Morales, M., Orosz, J. A., Shaw, J. S., et al. 2006, arXiv:astro-ph/ 0610225

López-Morales, M., \& Ribas, I. 2005, ApJ, 631, 1120

Mancini, L., Southworth, J., Ciceri, S., et al. 2014, A\&A, 562, A126

Mandel, K., \& Agol, E. 2002, ApJL, 580, L171

Mayor, M., Pepe, F., Queloz, D., et al. 2003, Msngr, 114, 20

Mohler-Fischer, M., Mancini, L., Hartman, J. D., et al. 2013, A\&A, 558, A55

Morales, J. C., Ribas, I., Jordi, C., et al. 2009, ApJ, 691, 1400

Nefs, S. V., Birkby, J. L., Snellen, I. A. G., et al. 2013, MNRAS, 431, 3240

Neves, V., Bonfils, X., Santos, N. C., et al. 2014, arXiv:1406.6127

Ofir, A., Gandolfi, D., Buchhave, L., et al. 2012, MNRAS, 423, L1

Pál, A. 2012, MNRAS, 421, 1825

Penev, K., Bakos, G. Á, Bayliss, D., et al. 2013, AJ, 145, 5

Popper, D. M. 1994, AJ, 108, 1091

Popper, D. M. 1997, AJ, 114, 1195

Queloz, D., Mayor, M., Udry, S., et al. 2001, Msngr, 105, 1

Rajpurohit, A. S., Reylé, C., Allard, F., et al. 2013, A\&A, 556, A15

Ribas, I. 2003, A\&A, 398, 239

Ribas, I. 2006, Ap\&SS, 304, 89

Sandquist, E. L., Mathieu, R. D., Brogaard, K., et al. 2013, ApJ, 762, 58

Schlafly, E. F., \& Finkbeiner, D. P. 2011, ApJ, 737, 103

Ségransan, D., Kervella, P., Forveille, T., \& Queloz, D. 2003, A\&A, 397, L5

Southworth, J. 2011, MNRAS, 417, 2166

Southworth, J. 2012, MNRAS, 426, 1291

Steffen, J. H., Fabrycky, D. C., Ford, E. B., et al. 2012, MNRAS, 421, 2342

ter Braak, C. J. F. 2006, Statistics and Computing, 16, 239

Torres, G. 2013, AN, 334, 4

Torres, G., Andersen, J., \& Giménez, A. 2010, A\&ARv, 18, 67

Torres, G., Bakos, G. Á, Kovács, G., et al. 2007, ApJL, 666, L121

Torres, G., Boden, A. F., Latham, D. W., Pan, M., \& Stefanik, R. P. 2002, AJ, 124,1716

Torres, G., \& Ribas, I. 2002, ApJ, 567, 1140

Triaud, A. H. M. J., Anderson, D. R., Collier Cameron, A., et al. 2013, A\&A, 551, A80

Triaud, A. H. M. J., Hebb, L., Anderson, D. R., et al. 2013, A\&A, 549, A18 Valenti, J. A., \& Fischer, D. A. 2005, ApJS, 159, 141

Winn, J. N. 2010, arXiv:1001.2010

Zhou, G., Bayliss, D., Hartman, J. D., et al. 2014, MNRAS, 437, 2831 